\title{
Classification of function spaces with the pointwise topology determined by a countable dense set
}

\author{
by
}

\author{
Tadeusz Dobrowolski (Norman, Okla.) and \\ Witold Marcis zew ski (Warszawa)
}

\begin{abstract}
We are concerned with $C_{A}(X)$, the space of continuous real valued functions on $X$ considered with the topology of pointwise convergence on $A$, where $A$ is a countable dense subset of $X$. We focus on the Borel and the topological classifications of the spaces $C_{A}(X)$. For example, we prove that for countable nondiscrete $X, C_{A}(X)$ is homeomorphic to $\sigma^{\omega}$, the countable product of $\sigma=\left\{\left(x_{i}\right) \in \mathbb{R}^{\omega} \mid x_{i}=0\right.$ a.e. $\}$, provided $C_{A}(X) \in F_{\sigma \delta}$.
\end{abstract}

1. Introduction. All spaces under consideration are completely regular. For a space $X, C_{\mathrm{p}}(X)$ denotes the space of all continuous real valued functions on $X$ with the pointwise convergence topology. If $A$ is a dense subset of $X$, then by $C_{A}(X)$ we denote the space of continuous real valued functions on $X$ with the topology of pointwise convergence on $A$. Hence, we have $C_{A}(X)=\{f|A| f$ is continuous on $X\} \subseteq \mathbb{R}^{A}$ and $C_{X}(X)=C_{\mathrm{p}}(X)$. Throughout this paper we will assume that $A$ is countable; consequently, $C_{A}(X)$ is a dense linear subspace of $\mathbb{R}^{A}$, a countable product of lines.

Recently, a lot of work has been done on the topological classification of Borel and projective function spaces $C_{\mathrm{p}}(X)$ for countable spaces $X$ (for references see $[\mathrm{DMM}]$ and $[\mathrm{CDM}])$. It has been shown that $C_{\mathrm{p}}(X)$, while Borel, is always of an exact multiplicative class $[\mathrm{CDM}]$ and it is conjectured that the topological and the Borel classifications coincide $[\mathrm{DMM}]$. For the countable spaces $X$ the spaces $C_{A}(X)$ seem to be a natural generalization

1991 Mathematics Subject Classification: Primary 46E10, 57N20, 54C35.

Key words and phrases: function space, topology determined by a countable set, borelian filters.

Research of the second author partially supported by KBN grant 211139101 .

The results of this paper were presented by the second author in January 1993 at the 21st Winter School on Abstract Analysis, Poděbrady, Czech Republic. 
of the spaces $C_{\mathrm{p}}(X)$. This paper is to initiate an investigation on the Borel complexity and the topological classification of the spaces $C_{A}(X)$. The Borel structure of the spaces $C_{A}(X)$ seems to be much more complicated than in the case of $C_{\mathrm{p}}(X)$. However, we are able to indicate some similarities between the topological classifications of the spaces $C_{A}(X)$ and $C_{\mathrm{p}}(X)$.

The spaces $C_{A}(X)$ also allow us to use some methods of descriptive set theory and infinite-dimensional topology (applicable for separable metrizable spaces) for the investigation of the function spaces on uncountable separable spaces. In this context the spaces $C_{A}(X)$ have appeared in the literature in several natural situations. Before we describe some of them we have to recall a few notions.

A map $f: M \rightarrow N$ between separable metrizable spaces is of the first Baire class if $f^{-1}(U)$ is an $F_{\sigma}$-subset of $M$ for every open $U \subset N$ (if $N$ is additionally a linear space then this means that $f$ is the pointwise limit of a sequence of continuous maps $M \rightarrow N)$. Let $P$ be the space of irrationals. It turns out that compact spaces that can be embedded in $B_{1}(P)$, the space of real valued first Baire class functions on $P$ with the topology of pointwise convergence, are of great importance in topology and Banach space theory (see [BFT] and [Ne, Section 1]); they are called Rosenthal compacta. The following result of Godefroy [Go, Theorem 4] shows how $C_{A}(X)$ spaces are involved in dealing with Rosenthal compacta.

1.1. A separable compact space $K$ is a Rosenthal compactum if and only if for every countable dense subset $A$ of $K$ the space $C_{A}(K)$ is analytic (i.e., a continuous image of $P$ ).

The problem of Borel classification of the spaces $C_{A}(K)$ for separable Rosenthal compact spaces $K$ has been disscussed in [Ma1].

Another important fact involving $C_{A}(X)$ spaces is the following factorization result [Ma1, Lemma 3.4]:

1.2. Let $X$ and $Y$ be separable spaces and $\varphi: C_{\mathrm{p}}(X) \rightarrow C_{\mathrm{p}}(Y)$ be a homeomorphism. For any countable sets $C \subseteq X$ and $D \subseteq Y$ there exist countable dense sets $A \subseteq X$ and $B \subseteq Y$ such that $C \subseteq A, D \subseteq B$ and the map $\pi_{B} \varphi \pi_{A}^{-1}: C_{A}(X) \rightarrow C_{B}(Y)$ is a homeomorphism $\left(\pi_{A}: C_{\mathrm{p}}(X) \rightarrow\right.$ $C_{A}(X)$ and $\pi_{B}: C_{\mathrm{p}}(Y) \rightarrow C_{B}(X)$ are the standard projections).

This result shows that the problem of the topological classification of the function spaces $C_{\mathrm{p}}(X)$ for separable spaces $X$ is related to the problem of the classification of the spaces $C_{A}(X)$.

Let us observe that the topology of $C_{A}(X)$ is precisely the weak topology on $C_{\mathrm{p}}(X)$ induced by the family of evaluation functionals at $a, a \in A$; this family is countable, consists of continuous linear functionals, and separates points of $C_{\mathrm{p}}(X)$. Any continuous linear functional on $C_{\mathrm{p}}(X)$ is a linear com- 
bination of finitely many evaluation functionals (at $x \in X$ ). The following example shows that the weak topology on $C_{\mathrm{p}}(X)$ induced by a countable family of continuous linear functionals which separate points of $C_{\mathrm{p}}(X)$ is not necessarily that of $C_{A}(X)$ for some countable dense $A \subseteq X$.

1.3. ExAmple. Let $\left\{q_{n}\right\}_{n=1}^{\infty}$ be an enumeration of the rationals in the line $\mathbb{R}$. The sequence $\left\{\varphi_{n}\right\}_{n=1}^{\infty}$ of continuous linear functionals on $C_{\mathrm{p}}(\mathbb{R})$ given by $\varphi_{n}(f)=f\left(q_{n}\right)+f\left(q_{n}+\pi / n\right), f \in C_{\mathrm{p}}(\mathbb{R}), n=1,2, \ldots$, separates points of $C_{\mathrm{p}}(\mathbb{R})$. However, every evaluation functional on $C_{\mathrm{p}}(\mathbb{R})$ is discontinuous in the weak topology induced by $\left\{\varphi_{n}\right\}_{n=1}^{\infty}$ on $C_{\mathrm{p}}(\mathbb{R})$.

Recall that if $M$ is a separable metrizable space and $\alpha$ is a countable ordinal then $\mathcal{A}_{\alpha}(M)$ (resp., $\mathcal{M}_{\alpha}(M)$ ) denotes the family of subsets of $M$ that are Borel of additive (resp., multiplicative) class $\alpha$. By $\mathcal{A}_{\alpha}$ (resp., $\mathcal{M}_{\alpha}$ ) we denote the class of spaces that are absolute Borel of additive (resp., multiplicative) class $\alpha$. If $A \in \mathcal{A}_{\alpha} \backslash \mathcal{M}_{\alpha}$ (resp., $\mathcal{M}_{\alpha} \backslash \mathcal{A}_{\alpha}$ or $\mathcal{A}_{\alpha} \cap \mathcal{M}_{\alpha} \backslash \bigcup_{\beta<\alpha}\left(\mathcal{A}_{\beta} \cup \mathcal{M}_{\beta}\right)$ ), then we say that $A$ is of the exact additive (resp., multiplicative or ambiguous) class $\alpha$. By $\mathcal{P}_{n}, n \geq 0$, we denote the $n$th projective class. A map $f: M \rightarrow N$ between separable metrizable spaces is Borel of class $\alpha$ if $f^{-1}(U) \in \mathcal{A}_{\alpha}(M)$ for every open $U \subset N$ (this means that $f$ is Borel of class $\alpha=1$ precisely when $f$ is of the first Baire class). The above terminology is that of [Kur].

In Section 2 we address some questions concerning the Borel (projective) structure of spaces $C_{A}(X)$. For instance, fixing $X$, we wonder how the Borel (projective) class of $C_{A}(X)$ will change when varying $A$. The following interesting question arises. What is the relationship between the exact Borel (projective) classes of $C_{\mathrm{p}}(X), C_{A}(X)$ and $C_{\mathrm{p}}(A)$ for countable $X$ ? For each countable ordinal $\alpha$, we provide an example of a countable space $X_{\alpha}$ and a dense subset $A_{\alpha}$ so that $C_{\mathrm{p}}\left(X_{\alpha}\right) \in \mathcal{M}_{2}$ and $C_{A_{\alpha}}\left(X_{\alpha}\right) \notin \mathcal{M}_{\alpha}$ (see 2.6).

In Section 3 we deal with spaces $X$ with exactly one nonisolated point. Such spaces can be identified with $\mathbb{N}_{F}=\mathbb{N} \cup\{\infty\}$ topologized by isolating the points of $\mathbb{N}=\{1,2, \ldots\}$ and using the family $\{A \cup\{\infty\} \mid A \in F\}$ as a neighborhood base at $\infty$, where $F$ is a filter on $\mathbb{N}$. In our considerations, if $F$ is a filter on a countable set $T$, then we usually identify $T$ and $\mathbb{N}$ and most often we let $T=\omega=\{0,1, \ldots\}$. We will always assume that $F$ contains the Fréchet filter $F_{0}$ consisting of all cofinite sets in $\omega$. We will write

$$
c_{F}=\left\{f \in \mathbb{R}^{\omega} \mid \forall(\varepsilon>0)\left(f^{-1}((-\varepsilon, \varepsilon)) \in F\right)\right\}
$$

and

$$
C_{F}=\left\{f \in \mathbb{R}^{\omega} \mid \exists(x \in \mathbb{R}) \forall(\varepsilon>0)\left(f^{-1}((x-\varepsilon, x+\varepsilon)) \in F\right)\right\} .
$$

It is known [Ma2, Lemma 2.1] that $c_{F}$ is (linearly) homeomorphic to $C_{\mathrm{p}}\left(\mathbb{N}_{F}\right)$. The space $C_{F}$ can be identified with $C_{\omega}\left(\mathbb{N}_{F}\right)$. Moreover, we have

$$
C_{F}=\left\{f \in \mathbb{R}^{\omega} \mid \forall(\varepsilon>0) \exists(A \in F) \forall\left(a_{1}, a_{2} \in A\right)\left(\left|f\left(a_{1}\right)-f\left(a_{2}\right)\right|<\varepsilon\right)\right\} .
$$


In these definitions the space $\omega$ can be replaced by any countable infinite set $T$. A filter $F$ on $T$ will be treated as a subset of $2^{T}$, a copy of the Cantor set. We address the following question. What is the relation between the Borel (projective) exact class of $F$ and that of $C_{F}$ ? Our answer is contained in 3.4 and is less satisfactory compared to the case of $c_{F}$ (see [DMM]).

Section 5 is devoted to the topological identification of $F_{\sigma \delta}$-spaces $C_{A}(X)$. The main result states that for countable nondiscrete $X$ and dense $A \subseteq X$, the space $C_{A}(X)$ is homeomorphic to $\sigma^{\omega}$ provided $C_{A}(X) \in F_{\sigma \delta}$; here $\sigma=\left\{\left(x_{n}\right) \in \mathbb{R}^{\omega} \mid x_{n}=0\right.$ a.e. $\}$. (This fact for $A=X$ is the main result of $[\mathrm{DMM}]$.) The same result holds for (not necessarily countable) Fréchet spaces $X$. Let us recall that $X$ is a Fréchet space if given a subset $Y \subset X$ and a point $x \in \bar{Y}$ there exists a sequence of elements of $Y$ that converges to $x$. The main tool to obtain these identification results is the method of absorbing sets (belonging to infinite-dimensional topology, see [BM]). An application of this technique to function spaces was initiated by van Mill in [vM]. We do not explicitly refer to this method; instead, we use some factorization facts and rely on results of [CDM]. However, we were not able to avoid another notion of infinite-dimensional topology: so-called $Z_{\sigma}$-spaces. We devote Section 4 to $Z_{\sigma}$-spaces $C_{A}(X)$. Many of our results concern also spaces $C_{A}^{*}(X)$, subsets of $C_{A}(X)$ consisting of all bounded functions.

In Section 6 we settle the case of metrizable $X$. It turns out that for a metrizable $X, C_{A}(X)$ is analytic only if $X$ is $\sigma$-compact (and then, it is homeomorphic to $\sigma^{\omega}$ ).

In the last section we provide examples of spaces $C_{F}$ with arbitrarily high Borel complexity. This is achieved by employing filters $F$ previously used in $[\mathrm{CDM}]$. Here, as in $[\mathrm{CDM}]$, not only do we show that for every $\alpha \geq 2$ there exists a filter $F$ such that $C_{F} \in \mathcal{M}_{\alpha} \backslash \mathcal{A}_{a}$, but actually it follows that $C_{F}$ is an absorbing set for the class of $\mathcal{M}_{\alpha}$. Hence, $C_{F}$ is homeomorphic to the standard, $\mathcal{M}_{\alpha}$-absorbing model $\Omega_{\alpha}$ (see $[\mathrm{BM}]$ ).

2. $C_{A}(X)$ versus $C_{B}(X)$. How much can they differ? For a space $X$ and countable dense subsets $A$ and $B$ of $X$, we will be interested in how much the Borel (projective) classes of $C_{A}(X)$ and $C_{B}(X)$ can differ. The following estimate was established in [Ma1, Theorem 2.2].

2.1. Proposition. Let $X$ be a Fréchet space and let $A, B \subseteq X$ be countable dense sets. For every countable ordinal $\alpha$ and every $n \in \omega$, we have:

(a) if $C_{A}(X) \in \mathcal{M}_{\alpha}$, then $C_{B}(X) \in \mathcal{M}_{1+\alpha}$,

(b) if $C_{A}(X) \in \mathcal{A}_{\alpha}$, then $C_{B}(X) \in \mathcal{A}_{1+\alpha}$,

(c) if $C_{A}(X) \in \mathcal{P}_{n}$, then $C_{B}(X) \in \mathcal{P}_{n}$. 
In particular, for infinite ordinals $\alpha$ and $n \in \omega$, the exact Borel (projective) classes of $C_{A}(X)$ and $C_{B}(X)$ coincide.

The following example shows that for non-Fréchet spaces $X$ the gap between Borel classes can be as big as we wish (for a space $Z, \beta Z$ denotes the Čech-Stone compactification of $Z$ ).

2.2. EXAMPLE. We have

(a) $C_{\omega}(\beta \omega) \in \mathcal{A}_{1} \backslash \mathcal{M}_{1}$ (actually, $C_{\omega}(\beta \omega)$ can be identified with the space $\Sigma=\left\{\left(x_{n}\right) \in \mathbb{R}^{\omega} \mid\left(x_{n}\right)\right.$ is bounded $\left.\}\right)$,

(b) if $p$ is an ultrafilter on $\omega$ (i.e., $p \in \beta \omega \backslash \omega$ ), then $C_{\omega \cup\{p\}}(\beta \omega) \notin \mathcal{P}_{1} \cup \mathcal{P}_{2}$ (actually, $C_{\omega \cup\{p\}}(\beta \omega)$ contains a closed copy of the ultrafilter $p$ ).

The statement of 2.2(b) can be reversed in the following way.

2.3. Proposition. Let $X$ be a compact space. Assume there are countable dense sets $A, B \subseteq X$ such that $C_{A}(X)$ is analytic and $C_{B}(X)$ is nonanalytic. Then $X$ contains a copy of $\beta \omega$.

Proof. Write $S=C_{A}(X)$ and consider $i: X \rightarrow \mathbb{R}^{S}$ defined by

$$
i(x)(f)=\arctan (f(x)), \quad f \in S, x \in X .
$$

It follows that $i$ is an embedding and $i(K)$ is norm-bounded (here, we consider the sup norm on the space of bounded functions on $S$ ). Moreover, for $a \in A, i(a)$ is continuous on $S$. Using the Godefroy characterization of Rosenthal compacta and our assumption, we get $i(K) \nsubseteq B_{1}(S)$. Now, our result is a consequence of the following statement from [P, p. 34] (see also $[\mathrm{BFT}]$ ): "For an arbitrary norm-bounded sequence $\left\{f_{j} \mid j \in \omega\right\}$ of continuous functions on $P$ (or, more generally, on an analytic space $X$ ) one and only one possibility occurs: either all pointwise accumulation points of $\left\{f_{j}\right\}$ are of first Baire class, or there exists a subsequence $\left\{f_{j} \mid j \in \omega\right\}$ which behaves on some Cantor set $T$ in $P$ like the sequence of projections $p_{j}: \omega^{\omega} \rightarrow\{0,1\}$ (in particular, in the second case, all accumulation points of $\left\{f_{j} \mid j \in \omega\right\}$ are non-Borel and the pointwise closure of the set $\left\{f_{j} \mid j \in \omega\right\}$ is homeomorphic to the Cech-Stone compactification of the natural numbers".

To get our assertion, enumerate $A=\left\{a_{j} \mid j \in \omega\right\}$ and apply the above statement to the sequence $\left\{i\left(a_{j}\right) \mid j \in \omega\right\}$.

The following result is a direct consequence of 2.3 and 1.1.

2.4. Corollary. Let $X$ be a compact space and let $A$ be a countable dense subset of $X$. If $C_{A}(X)$ is analytic then either $X$ is a Rosenthal compactum or else $X$ contains a copy of $\beta \omega$.

It has been shown [CDM, Theorem 5.1] that for every countable space $X$, the space $C_{\mathrm{p}}(X)$, when Borel, is of an exact multiplicative class. In connection with this and 2.2(a) we ask: 
2.5. Question. Is there an example of a (countable) space $X$ such that $C_{A}(X) \in \mathcal{A}_{\alpha} \backslash \mathcal{M}_{\alpha}, \alpha>1$, for some countable dense set $A \subseteq X$ ?

Next we show that the gap between Borel classes of $C_{\mathrm{p}}(X)=C_{X}(X)$ and $C_{A}(X)$ can be large even for countable $X$.

2.6. Proposition. For every countable ordinal $\alpha$, there exists a countable space $X_{\alpha}$ and a dense set $A \subseteq X_{\alpha}$ such that $C_{\mathrm{p}}\left(X_{\alpha}\right) \in \mathcal{M}_{2}$ and $C_{A}\left(X_{\alpha}\right) \notin \mathcal{M}_{\alpha}$.

Proof. We will use induction on $\alpha$. Set $X_{0}=A=\omega+1$. It is obvious that $C_{\mathrm{p}}\left(X_{0}\right) \in \mathcal{M}_{2}$; by [DGM, Corollary 1.2], $C_{\mathrm{p}}\left(X_{0}\right)$ is homeomorphic to $\sigma^{\omega}$ and hence it belongs to $\mathcal{M}_{2} \backslash \mathcal{A}_{2}$. Let us distinguish $x_{0}=\omega \in X_{0}$ and $B_{0}=\omega$; note that $B_{0}$ is the set of all isolated points of $X_{0}$ and $A=B_{0} \cup\left\{x_{0}\right\}$.

Suppose the spaces $X_{\beta}$ have been constructed (for all $\beta<\alpha$ ), sets $B_{\beta}$ of all isolated points of $X_{\beta}$ and points $x_{\beta} \in X_{\beta} \backslash B_{\beta}$ have been determined so that $A_{\beta}=B_{\beta} \cup\left\{x_{\beta}\right\}$ is a countable dense subset of $X_{\beta}$ so that $C_{A_{\beta}}\left(X_{\beta}\right) \notin$ $\mathcal{M}_{\alpha}$ and $C_{\mathrm{p}}\left(X_{\beta}\right) \in \mathcal{M}_{2}$. For every $n \in \omega$, let $\alpha_{n}=\beta$ provided $\alpha=\beta+1$. If $\alpha$ is a limit ordinal, fix a sequence of ordinals $\left\{\alpha_{n}\right\}_{n \in \omega}$ with $\alpha_{n}<\alpha, n \in \omega$, and $\sup _{n} \alpha_{n}=\alpha$. Form a direct sum $Y_{\alpha}=\left(\bigcup_{n \in \omega}\{n\} \times X_{\alpha_{n}}\right) \oplus(\omega+1)$. Consider an equivalence relation $R$ on $Y_{\alpha}$ which identifies $\left(n, x_{\alpha_{n}}\right)$ with $n \in \omega+1$. We set $X_{\alpha}=Y_{\alpha} / R$ to be the quotient space. Write $q: Y_{\alpha} \rightarrow X_{\alpha}$ for the quotient map. We let

$$
B_{\alpha}=\left\{x \in X_{\alpha} \mid x \text { is isolated in } X_{\alpha}\right\}
$$

and $x_{\alpha}=q(\omega)$, where $\omega \in \omega+1$. By the construction $B_{\alpha}=\bigcup_{n \in \omega} q(\{n\} \times$ $\left.B_{\alpha_{n}}\right)$. Finally, we let $A_{\alpha}=B_{\alpha} \cup\left\{x_{\alpha}\right\}$ and observe that $A_{\alpha}$ is countable and dense in $X_{\alpha}$. (Note that $X_{1}$ and $A_{1}$ are the Arens spaces described in Examples 1.6.19 and 1.6.20 of [En], respectively).

Observe that $f$ is continuous on $X_{\alpha}$ if and only if $f \mid q\left(\{n\} \times X_{\alpha_{n}}\right)$ and $f \mid q(\omega+1), n \in \omega$, are continuous. It then inductively follows that $C_{\mathrm{p}}\left(X_{a}\right) \in$ $\mathcal{M}_{2}$. We now show that $C_{A_{\alpha}}\left(X_{\alpha}\right) \notin \mathcal{M}_{\alpha}$. Consider the following subspaces $E_{\alpha}$ and $F_{\alpha}$ of $C_{B_{\alpha}}\left(X_{\alpha}\right)$ :

$$
\begin{aligned}
& E_{\alpha}=\left\{f \in C_{B_{\alpha}}\left(X_{\alpha}\right) \cap\{0,1\}^{B_{\alpha}} \mid f\left(x_{\alpha}\right)=0\right\}, \\
& F_{\alpha}=\left\{f \in C_{B_{\alpha}}\left(X_{\alpha}\right) \cap\{0,1\}^{B_{\alpha}} \mid f\left(x_{\alpha}\right)=1\right\} .
\end{aligned}
$$

Claim. Write $G_{\alpha}=E_{\alpha} \cup F_{\alpha}$. The pairs $\left(G_{\alpha}, E_{\alpha}\right)$ and $\left(G_{\alpha}, F_{\alpha}\right)$ are Wadge $\left(2^{\omega}, \mathcal{A}_{\alpha} \cup \mathcal{M}_{\alpha}\left(2^{\omega}\right)\right)$-complete (i.e., for every $C \in \mathcal{A}_{\alpha} \cup \mathcal{M}_{\alpha}\left(2^{\omega}\right)$ there exists a map $\varphi: 2^{\omega} \rightarrow G_{\alpha}$ so that $\varphi^{-1}\left(E_{\alpha}\right)=C$ or $\varphi^{-1}\left(F_{\alpha}\right)=C$, respectively).

We will provide an inductive proof. If $\alpha=0$, then $E_{0}$ and $F_{0}$ are copies of the rationals and both $E_{0}$ and $F_{0}$ are dense in $G_{0}$. Let $C \in \mathcal{M}_{0}\left(2^{\omega}\right)$ (i.e., $C$ is a closed subset of $\left.2^{\omega}\right)$. Pick a sequence $\left\{x_{n}\right\}_{n=1}^{\infty} \subset E_{0}$ such that $\lim x_{n}=$ $x_{0} \in F_{0}$. Find a map $\varphi: 2^{\omega} \rightarrow\left\{x_{n} \mid n \in \omega\right\}$ such that $\varphi^{-1}\left(\left\{x_{0}\right\}\right)=C$. If 
$C \in \mathcal{A}_{0}\left(2^{\omega}\right)$ (i.e., $C$ is open in $2^{\omega}$ ), apply the same argument interchanging the roles of $E_{0}$ and $F_{0}$. Let $C \in \mathcal{A}_{\alpha}$ and pick $C_{n} \in \mathcal{M}_{\alpha_{n}}, C_{n} \subseteq C_{n+1}$, $n \in \omega$, so that $C=\bigcup_{n \in \omega} C_{n}$. We have $E_{\alpha}=\mathbb{F P}\left(G_{\alpha_{n}}, E_{\alpha_{n}}\right)$ and $F_{\alpha}=$ $\mathbb{F} \mathbb{P}\left(G_{\alpha_{n}}, F_{\alpha_{n}}\right)$, where for a sequence $\left\{\left(X_{n}, A_{n}\right)\right\}_{n \in \omega}$ of pairs of spaces

$$
\mathbb{F P}\left(X_{n}, A_{n}\right)=\left\{\left(x_{n}\right) \in \prod_{n \in \omega} X_{n} \mid x_{n} \in A_{n} \text { a.e. }\right\}
$$

(notation of [CDM, Section 8]). Using the inductive assumption, we can find $\varphi_{n}: 2^{\omega} \rightarrow G_{\alpha_{n}} \subset \mathbb{R}^{B_{\alpha_{n}}}$ such that $\varphi_{n}^{-1}\left(E_{\alpha_{n}}\right)=C_{n}$. Take $\varphi=\Delta \varphi_{n}$ : $2^{\omega} \rightarrow \mathbb{R}^{\cup B_{\alpha_{n}}}$ given by $\varphi(p)=\left(\varphi_{n}(p)\right)$ and observe that $\varphi\left(\bigcup_{n \in \omega} C_{n}\right) \subset$ $\mathbb{F P}\left(G_{\alpha_{n}}, E_{\alpha_{n}}\right)$ and $\varphi\left(2^{\omega} \backslash \bigcup_{n \in \omega} C_{n}\right) \subset \prod_{n \in \omega} F_{\alpha_{n}} \subset F_{\alpha}$ (cf. [CDM, Proof of 8.1]). This settles the case where $C \in \mathcal{A}_{\alpha}$. By symmetry the same argument works for $C \in \mathcal{M}_{\alpha}$.

An easy application of the Claim yields $E_{\alpha} \notin \mathcal{M}_{\alpha} \cup \mathcal{A}_{\alpha}$. Since $E_{\alpha}$ can be identified with the set

$$
\left\{f \in C_{A_{\alpha}}\left(X_{\alpha}\right) \cap\{0,1\}^{A_{\alpha}} \mid f\left(x_{\alpha}\right)=0\right\},
$$

a closed subset of $C_{A_{\alpha}}\left(X_{\alpha}\right)$, our assertion follows.

2.7. R e mark. Let us note that even for a compact $X$ the Borel class of $C_{A}(X)$ can be as high as we wish. Such spaces $X$ can be taken as Rosenthal compacta and were provided in [Ma1]. Let us indicate another way of finding such $X$. By [LvMP, Theorem 4.1], there exists a countable regular space $A$ with exactly one nonisolated point such that the Borel class of $C_{\mathrm{p}}(A)$ (and hence, of $\left.C_{\mathrm{p}}^{*}(A)\right)$ is as high as we wish. Let $X=\beta A$ and observe that $C_{A}(X)=C_{\mathrm{p}}^{*}(A)$.

2.8. R e mark. Consider $X=\mathbb{N}_{F}$ for a filter $F$ on $\omega$. Applying 3.3, we see that $C_{\omega}\left(\mathbb{N}_{F}\right)=C_{F}$ contains a closed copy of $F$; and hence can have as complicated Borel structure as we wish. At the same time $C_{\mathrm{p}}(\omega)=\mathbb{R}^{\omega}$ $\in \mathcal{M}_{1}$.

The statements 2.6 and 2.8 show that, within the Borel hierarchy, the Borel class of $C_{A}(X)$ cannot be estimated by either the class of $C_{\mathrm{p}}(X)$ or $C_{\mathrm{p}}(A)$.

2.9. Question. Let $X$ be a countable space and let $A$ be a dense subset of $X$. Is the Borel (projective) class of $C_{\mathrm{p}}(X)$ (resp., $\left.C_{\mathrm{p}}(A)\right)$ determined by that of $C_{A}(X)$ ? Is $C_{\mathrm{p}}(X)$ Borel (analytic) if $C_{A}(X)$ is? Can the exact Borel (projective) class of $C_{\mathrm{p}}(X)$ be greater than that of $C_{A}(X)$ ?

2.10. R e mark. Let $\pi_{A}: C_{\mathrm{p}}(X) \rightarrow C_{A}(X)$ be the projection map. Since $C_{A}(X)=\pi_{A}\left(C_{\mathrm{p}}(X)\right)$ and $\pi_{A}$ is injective, $C_{A}(X)$ is Borel provided $C_{\mathrm{p}}(X)$ is (see [Kur]). We claim that also $C_{\mathrm{p}}(A)$ is analytic provided $C_{A}(X)$ is. The latter can be shown as follows. If $C_{A}(X)$ is analytic, then for every accumulation point $a \in A$ the filter $F_{a}=\left\{Y \cap A \mid Y \in 2^{X \backslash\{a\}}\right.$ and $\left.a \in \operatorname{Int}(Y \cup\{a\})\right\}$ 
is an analytic filter on $A \backslash\{a\}$ (cf. the proof of [DMM, Corollary 3.6]). If now by $A_{F_{a}}$ we denote the space $A$ topologized by isolating the points of $A \backslash\{a\}$ and by using the family $\left\{A \cup\{a\} \mid A \in F_{a}\right\}$ as neighborhood base at $a$, we have $C_{\mathrm{p}}(A)=\bigcap\left\{C_{\mathrm{p}}\left(A_{F_{a}}\right) \mid a\right.$ is an accumulation point of $\left.A\right\}$. Since each $C_{\mathrm{p}}\left(A_{F_{a}}\right)$ is analytic, so is $C_{\mathrm{p}}(A)$ (this argument was taken from [DMM, Lemma 4.3]).

Let us notice that the last two questions of 2.9 have negative answers for bounded function spaces.

2.11. ExAmple. For any ultrafilter $p \in \beta \omega \backslash \omega$ we have, $C_{\omega}^{*}(\omega \cup\{p\})=$ $\Sigma \in \mathcal{A}_{1} \backslash \mathcal{M}_{1}$ and $C_{\mathrm{p}}^{*}(\omega \cup\{p\}) \notin \mathcal{P}_{1} \cup \mathcal{P}_{2}$.

Observe that, for every $p$ from Example 2.11, we also have $C_{\omega}(\omega \cup\{p\}) \notin$ $\mathcal{P}_{1} \cup \mathcal{P}_{2}$. Hence, we see that the gap between Borel classes of $C_{A}^{*}(X)$ and $C_{A}(X)$ can be as big as we wish.

3. Borel type of spaces $C_{F}$. For the Fréchet filter $F_{0}$ on $\omega$ we use the classical functional analysis symbols $c_{0}$ and $c$ to denote the spaces $c_{F_{0}}$ and $C_{F_{0}}$. The spaces $c_{0}$ and $c$ considered as Banach spaces (with the sup norm) are linearly isomorphic. The following fact shows a dramatic difference if one considers $c_{0}$ and $c$ as subspaces of $\mathbb{R}^{\omega}$.

3.1. Proposition. The spaces $c_{0}, c \subset \mathbb{R}^{\omega}$ are not linearly isomorphic.

Proof. Assume $T: c_{0} \rightarrow c$ establishes a linear topological isomorphism of $c_{0}$ onto $c$. Let $\|\cdot\|$ be the sup-norm on $c$ (and $c_{0}$ ). First we note that the graph of $T$,

$$
\Gamma=\left\{(x, T x) \mid x \in c_{0}\right\} \subset c_{0} \times c,
$$

is closed in the norm topology. This follows from the obvious fact that $\Gamma$ is closed with respect to the coordinatewise topology on $c_{0} \times c$ and the fact that the latter topology is weaker than the norm topology on $c_{0} \times c$. Consequently, by the Closed Graph Theorem, $T$ establishes a linear isomorphism of $\left(c_{0},\|\cdot\|\right)$ onto $(c,\|\cdot\|)$.

Consider the continuous linear functional $\ell:(c,\|\cdot\|) \rightarrow \mathbb{R}$ given by $\ell\left(\left(x_{n}\right)\right)=\lim x_{n}$. It follows that $\ell \circ T:\left(c_{0},\|\cdot\|\right) \rightarrow \mathbb{R}$ is continuous and $\ell \circ T \not \equiv$ 0 . This obviously implies that $\ell \circ T$ is continuous with respect to the weak topology $\omega$ on $c_{0}$. Consequently, for every convex closed neighborhood $U$ of $0 \in\left(c_{0},\|\cdot\|\right), \operatorname{ker}(\ell \circ T) \cap U$ is closed in the $\omega$-topology, and $\operatorname{ker}(\ell \circ T) \cap U \neq U$.

Let $B=\{y \in c \mid\|y\| \leq 1\}$ be the unit ball in $c$. We will show that for $U=T^{-1}(B), \operatorname{ker}(\ell \circ T) \cap U$ is dense in $U$ in the $\omega$-topology, contradicting the above fact. To this end, first notice that given $y \in B$, there exists a sequence $\left\{y_{n}\right\}_{n \in \omega} \subset B \cap c_{0}$ so that $\left\{y_{n}\right\}_{n \in \omega}$ converges to $y$ in $\mathbb{R}^{\omega}$. We see that $\left\{T^{-1} y_{n}\right\}_{n \in \omega}$ converges to $T^{-1} y$ in $\mathbb{R}^{\omega}$ and $T^{-1} y_{n} \in \operatorname{ker}(\ell \circ T)$. Since 
the $\omega$-topology coincides with the $\mathbb{R}^{\omega}$-topology on bounded sets, it follows that $\left\{T^{-1} y_{n}\right\}$ converges to $T^{-1} y$ in the $\omega$-topology.

Before we enter a discussion on Borel types of $C_{F}$, let us formulate the following general fact which will be employed later on; its proof can be obtained easily by using the argument of the proof of [Ma2, Lemma 2.1].

3.2. Lemma. Let $F$ be a filter on $\omega$ which is not the Fréchet filter. Then $C_{F}$ is homeomorphic to the product $C_{F} \times \mathbb{R}^{\omega}$.

We identify a filter $F$ on a countable set $T$ with a subset of a Cantor set $2^{T}$. As shown in [DMM, Lemma 4.2] the exact Borel class of $c_{F}$ is entirely determined by that of $F$ in $2^{T}$. Here we try to recover this result for the space $C_{F}$. The first result shows that Borel (projective) classes of $C_{F}$ and $C_{F}^{*}$ are not lower than that of $F$.

3.3. Proposition. Let $F$ be a filter on a countable set T. For every countable ordinal $\alpha$ and $n \in \omega$, we have:

(a) if $C_{F} \in \mathcal{M}_{\alpha}$, then $F \in \mathcal{M}_{\alpha}$,

(b) if $C_{F} \in \mathcal{A}_{\alpha}$, then $F \in \mathcal{A}_{\alpha}$,

(c) if $C_{F} \in \mathcal{P}_{n}$, then $F \in \mathcal{P}_{n}$.

In (a)-(c), the space $C_{F}$ can be replaced by $C_{F}^{*}$ provided $F$ is not an ultrafilter.

Proof. We may assume $T=\omega$. Consider

$$
Z=\left\{f \in C_{F} \mid \forall(n \in \omega)(f(n)=0 \text { or } f(n)=n+1)\right\},
$$

a closed subset of $C_{F}$. The map $\left(x_{n}\right) \rightarrow\left((n+1)\left(1-x_{n}\right)\right)$ embeds $2^{\omega}$ in $\mathbb{R}^{\omega}$ and sends $F$ onto $Z$. Thus, the $C_{F}$-part of our assertion follows.

Assume that $F$ is not an ultrafilter on $\omega$ (see 2.11). There exists a subset $M \subset \omega$ such that neither $M$ nor $N=\omega \backslash M$ belongs to $F$. Consider the filters

$$
G=\{A \cap M \mid A \in F\} \quad \text { and } \quad H=\{A \cap H \mid A \in F\}
$$

induced by $F$ on $M$ and $N$, respectively. The sets

$$
\begin{aligned}
& Y_{1}=\left\{f \in C_{F}^{*} \cap\{0,1\}^{\omega}|f| N=0\right\}, \\
& Y_{2}=\left\{f \in C_{F}^{*} \cap\{0,1\}^{\omega}|f| M=0\right\}
\end{aligned}
$$

are closed subsets of $C_{F}^{*}$. It is clear that $G$ and $H$ are homeomorphic to $Y_{1}$ and $Y_{2}$, respectively. Finally, since $F$ can be identified with the product $G \times H$, our assertion follows.

Here is our counterpart of [DMM, Lemma 4.2] for spaces $C_{F}$.

3.4. Proposition. Let $F$ be a filter on $\omega$. For every countable ordinal $\alpha \geq 1$ and $n \in \omega$, we have: 
(a) if $F \in \mathcal{A}_{\alpha}$, then $C_{F} \in \mathcal{M}_{\alpha+1}$,

(b) if $F \in \mathcal{M}_{\alpha}$, then $C_{F} \in \mathcal{M}_{\alpha+1} \cap \mathcal{A}_{\alpha+1}$ (more exactly, $C_{F}$ is a difference of two sets belonging to $\mathcal{M}_{\alpha}$ ),

(c) if $F \in \mathcal{P}_{n}$, then $C_{F} \in \mathcal{P}_{n}$.

Proof. First we provide an argument which shows (a) and (c). Then we adapt this argument to check that whenever $F \in \mathcal{M}_{\alpha}$ then $C_{F} \cap[0,1]^{\omega} \in$ $\mathcal{M}_{\alpha}$. Finally, since $C_{F}$ is homeomorphic to

$$
\begin{aligned}
\left\{f \in C_{F} \mid \forall(n \in \omega)(f(n)\right. & \left.\left.\in(0,1) \text { and } \lim _{F} f(n) \in(0,1)\right)\right\} \\
& =\left(C_{F} \cap[0,1]^{\omega}\right) \cap(0,1)^{\omega} \backslash\left(c_{F} \cup\left(1+c_{F}\right)\right),
\end{aligned}
$$

the space $C_{F}$ is a difference of two sets belonging to $\mathcal{M}_{\alpha}$ (by [DMM, Lemma 4.2], $c_{F}$ and hence $1+c_{F}=\left\{\left(x_{n}+1\right) \in \mathbb{R}^{\omega} \mid\left(x_{n}\right) \in c_{F}\right\}$ belong to $\left.\mathcal{M}_{\alpha}\right)$.

Assume $F \in \mathcal{A}_{\alpha}$ (resp., $F \in \mathcal{P}_{n}$ ). For $n \in \omega$ and $k \in \mathbb{Z}$, write

$$
T_{n, k}=\left[\frac{2 k}{2 n+2}, \frac{2 k+1}{2 n+2}\right] \text {. }
$$

Set $T_{n}=\bigcup_{k \in \mathbb{Z}} T_{n, k}$. For $m \in \mathbb{N}$ and $l \in \mathbb{Z}$, define

$$
S_{m, l, n}=\bigcup_{k}\left\{T_{n, k} \mid \operatorname{dist}\left(\frac{l}{m}, T_{n, k}\right) \leq \frac{1}{m}\right\} .
$$

Let $\varrho_{m, l}: \prod_{n \in \omega} T_{n} \rightarrow 2^{\omega}$ be given by

$$
\varrho_{m, l}(f)(n)= \begin{cases}1 & \text { if } f(n) \in S_{m, l, n} \\ 0 & \text { otherwise }\end{cases}
$$

for $f \in \prod_{n \in \omega} T_{n}$. One can check that $\varrho_{m, l}$ is continuous. We claim that

$$
C_{F} \cap \prod_{n \in \omega} T_{n}=\bigcap_{m=1}^{\infty} \bigcup_{l \in \mathbb{Z}} \varrho_{m, l}^{-1}(F) .
$$

(If $f=(f(n)) \in \prod_{n \in \omega} T_{n}$ and there exists $x \in \mathbb{R}$ such that for all $\varepsilon>0$ we have $f^{-1}((x-\varepsilon, x+\varepsilon)) \in F$, then for every $m \geq 1$ one can find $l \in \mathbb{Z}$ so that $|x-l / m| \leq 1 /(2 m)$ and check that $\left\{n \mid f(n) \in S_{m, l, n}\right\} \in F$; this shows $\varrho_{m, l}(f) \in F$. Conversely, if for every $m \geq 1$ one can find $l \in \mathbb{Z}$ so that $\left\{n \mid f(n) \in S_{m, l, n}\right\} \in F$, then there exists $A \in F$ so that whenever $i, j \in A$ then $|f(i)-f(j)|<4 / m$; this implies $f \in C_{F}$.)

Pick a map $\psi_{n}: T_{n} \rightarrow \mathbb{R}$ that transforms $T_{n, k}$ affinely onto $\left[\frac{2 k}{2 n+2}, \frac{2 k+2}{2 n+2}\right]$, $k \in \mathbb{Z}$. Letting $\psi=\prod_{n \in \omega} \psi_{n}: \prod_{n \in \omega} T_{n} \rightarrow \mathbb{R}^{\omega}$ we see that $\psi$ is a perfect map and that

$$
C_{F} \cap \prod_{n \in \omega} T_{n}=\psi^{-1}\left(C_{F}\right) .
$$

Applying (1), $C_{F} \cap \prod_{n \in \omega} T_{n} \in \mathcal{M}_{\alpha+1}$ (resp., $C_{F} \cap \prod_{n \in \omega} T_{n} \in \mathcal{P}_{n}$ ). By (2) and a result of Saint Raymond [SR1], $C_{F} \in \mathcal{M}_{\alpha+1}$ (resp., $C_{F} \in \mathcal{P}_{n}$ ). 
Now, let $F \in \mathcal{M}_{\alpha}$. We must show that $C_{F} \cap[0,1]^{\omega} \in \mathcal{M}_{\alpha}$. Using the sets $T_{n, k}$ we define

$$
T_{n}=\bigcup_{k=0}^{n-1} T_{n, k}
$$

With these $T_{n}$, as previously, define for $m \in \mathbb{N}$ and $l \in\{0, \ldots, m\}$ the sets $S_{m, l, n}$ and maps $\varrho_{m, l}$. One can check

$$
C_{F} \cap \prod_{n \in \omega} T_{n}=\bigcap_{m=1}^{\infty} \bigcup_{l=0}^{m} \varrho_{m, l}^{-1}(F) .
$$

Let $\psi_{n}: T_{n} \rightarrow[0,1]$ be a map such that $T_{n, k}$ is affinely transformed onto $\left[\frac{2 k}{2 n+2}, \frac{2 k+2}{2 n+2}\right]$. Letting $\psi=\prod_{n \in \omega} \psi_{n}: \prod_{n \in \omega} T_{n} \rightarrow[0,1]^{\omega}$, we have

$$
C_{F} \cap \prod_{n \in \omega} T_{n}=\psi^{-1}\left(C_{F} \cap[0,1]^{\omega}\right) .
$$

Using (1)' $C_{F} \cap \prod_{n \in \omega} T_{n} \in \mathcal{M}_{\alpha}$. As above, by (2)' and a result of Saint Raymond [SR1], $C_{F} \cap[0,1]^{\omega} \in \mathcal{M}_{\alpha}$.

3.5. Question. Let $F \in \mathcal{M}_{\alpha}$ be a filter on $\omega$. Is $C_{F} \in \mathcal{M}_{\alpha}$ ?

Let $F$ be a filter on $\omega$. Identify the space $C_{F}$ with $C_{\omega}\left(\mathbb{N}_{F}\right)$. Using this identification, let $\ell: C_{F} \rightarrow \mathbb{R}$ be given by $\ell(f)=f(\infty)$. We see that $\ell$ is well defined and can be viewed as $\ell(f)=\lim _{F} f(n)$.

3.6. Proposition. For a filter $F \in \mathcal{A}_{\alpha} \cup \mathcal{M}_{\alpha}$, and a countable ordinal $\alpha, \alpha \geq 1, \ell: C_{F} \rightarrow \mathbb{R}$ is Borel of class $\alpha$.

Proof. Let $U \subseteq \mathbb{R}$ be an open set. We must show that $\ell^{-1}(U) \in$ $\mathcal{A}_{\alpha}\left(C_{F}\right)$.

We shall use some of the notation employed in the proof of 3.4. For $n \in \omega$ and $m \in \mathbb{N}$, let

$$
P_{n, m}=\bigcup\left\{T_{n, k} \mid \forall\left(t \in T_{n, k}\right)(\operatorname{dist}(t, \mathbb{R} \backslash U)>1 / m)\right\} .
$$

Recall that $T_{n}=\bigcup_{k \in \mathbb{Z}} T_{n, k}$ and define $\zeta_{m}: \prod_{n \in \omega} T_{n} \rightarrow\{0,1\}^{\omega}$ by letting for $f \in \prod_{n \in \omega} T_{n}$,

$$
\zeta_{m}(f)(n)= \begin{cases}1 & \text { if } f(n) \in P_{n, m}, \\ 0 & \text { otherwise. }\end{cases}
$$

Clearly, $\zeta_{m}$ is continuous, $m \geq 1$. Let $\psi: \prod_{n \in \omega} T_{n} \rightarrow \mathbb{R}^{\omega}$ be that of the proof of 3.4 .

Suppose $F \in \mathcal{A}_{\alpha}$. Consider $S=\bigcup_{m=1}^{\infty} \zeta_{m}^{-1}(F)$. We see that $S \in \mathcal{A}_{\alpha}(S \in$ $\mathcal{A}_{1}\left(\prod_{n \in \omega} T_{n}\right)$ for $\left.\alpha=1\right)$. We claim that $\psi^{-1}(\psi(S))=S$. Let $f, g \in \prod_{n \in \omega} T_{n}$ 
be such that $g \in \bigcup_{m=1}^{\infty} \zeta_{m}^{-1}(F)$ and $\psi(f)=\psi(g)$. Since $\psi(f)=\psi(g)$, we have $|g(n)-f(n)|<1 / n$. Take $m_{0}$ such that $g \in \zeta_{m_{0}}^{-1}(F)$. If $n>2 m_{0}$ and $g(n) \in P_{n, m_{0}}$, then $f(n) \in P_{n, 2 m_{0}}$. It follows that $\zeta_{2 m_{0}}(f) \supset \zeta_{m_{0}}(g) \backslash$ $\left\{0,1, \ldots, 2 m_{0}\right\}$; and hence $\zeta_{2 m_{0}}(f) \in F$. This shows that $f \in \zeta_{2 m_{0}}^{-1}(F) \subset S$.

Suppose $F \in \mathcal{M}_{\alpha}$. Let $\mathcal{J}=\{A \subset \omega \mid \omega \backslash A \in F\}$ be the dual ideal of $F$; $\mathcal{J}$ is homeomorphic to $F$. Consider $T=\left(\prod_{n \in \omega} T_{n}\right) \backslash \bigcap_{m=1}^{\infty} \zeta_{n}^{-1}(\mathcal{J})$. We see that $T \in \mathcal{A}_{\alpha}$. We claim that $\psi^{-1}(\psi(T))=T$. Let $f, g \in \prod_{n \in \omega} T_{n}$ be such that $f \notin \bigcap_{m=1}^{\infty} \zeta_{m}^{-1}(\mathcal{J})$ and $\psi(f)=\psi(g)$. Take $m_{0}$ such that $f \notin \zeta_{m_{0}}^{-1}(\mathcal{J})$. If $n>2 m_{0}$ and $f(n) \in P_{n, m_{0}}$, then $g(n) \in P_{n, 2 m_{0}}$. As previously, it follows that $\zeta_{2 m_{0}}(g) \notin \mathcal{J}$; hence $g \in T$.

We now claim that

$$
C_{F} \cap S=C_{F} \cap T=\left(\prod_{n \in \omega} T_{n}\right) \cap \ell^{-1}(U) .
$$

Namely, we have $f \in S$ (resp., $f \in T$ ) if and only if there exists $m \geq 1$ such that $f \in \zeta_{m}^{-1}(F)$ (resp., $f \notin \zeta_{m}^{-1}(\mathcal{J})$ ). Suppose $f \in\left(\prod_{n \in \omega} T_{n}\right) \cap \ell^{-1}(U)$ and $f(\infty)=x \in U$. Let $\varepsilon=\operatorname{dist}(x, \mathbb{R} \backslash U)$. For $m, n>4 / \varepsilon$ we have $(x-\varepsilon / 2, x+\varepsilon / 2) \cap T_{n} \subseteq P_{n, m}$. It follows that $f \in \zeta_{m}^{-1}(F)$ and hence $f \notin \zeta_{m}^{-1}(\mathcal{J})$. Suppose now that $f \in C_{F}$ and $f \in \zeta_{m}^{-1}(F)$. Since $f \notin \zeta_{m}^{-1}(\mathcal{J})$ the set $R=\left\{n \mid f(n) \in P_{n, m}\right\}$ is not in $\mathcal{J}$. Take $x \notin U$. Then for every $n \geq 1,(x-1 /(2 m), x+1 /(2 m)) \cap P_{n, m}=\emptyset$. Therefore for $Q=\{n \mid f(n) \in$ $(x-1 /(2 m), x+1 /(2 m))\}$ we have $R \cap Q=\emptyset$. Since $R \notin \mathcal{J}$, we have $Q \notin F$. It follows that $f(\infty) \neq x$, so $f(\infty) \in U$.

Since $\lim _{n}(f(n)-\psi(f)(n))=0$, one sees that $\lim _{F} f(n)=\lim _{F} \psi(f)(n)$; and consequently

$$
\ell^{-1}(U)=\psi\left(\left(\prod_{n \in \omega} T_{n}\right) \cap \ell^{-1}(U)\right) .
$$

This together with (1) and the fact that $\psi^{-1}\left(C_{F}\right)=C_{F} \cap \prod_{n \in \omega} T_{n}$ yields

$$
\begin{aligned}
& \ell^{-1}(U)=\psi\left(C_{F} \cap S\right)=C_{F} \cap \psi(S), \\
& \ell^{-1}(U)=\psi\left(C_{F} \cap T\right)=C_{F} \cap \psi(T) .
\end{aligned}
$$

The facts that $\psi^{-1}(\psi(S))=S$ and $\psi^{-1}(\psi(T))=T$ together with a result of Saint Raymond [SR1] show that, under our assumption, $C_{F} \cap \varphi(S)$ and $C_{F} \cap \varphi(T)$ belong to $\mathcal{A}_{\alpha}\left(C_{F}\right)$.

The following is a partial answer to Question 3.5.

3.7. Corollary. For $n \in \omega$, let $F_{n}$ be a filter on $\omega$ with $F_{n} \in \bigcup_{\beta<\alpha} \mathcal{A}_{\beta}$, $\alpha \geq 1$. Then for the filter $F=\prod_{n \in \omega} F_{n}$ on $\omega \times \omega$, we have $C_{F} \in \mathcal{M}_{\alpha}$.

Pro of. We obviously have

$$
F=\left\{A \subset \omega \times \omega \mid \forall(n \in \omega)\left(\{m \mid(m, n) \in A\} \in F_{n}\right)\right\} .
$$


Moreover, for $f=(f(m, n)) \in \mathbb{R}^{\omega \times \omega}, f \in C_{F}$ if and only if $f(\cdot, n) \in C_{F_{n}}$, $n \in \omega$, and $\lim _{F_{n}} f(\cdot, n)=\lim _{F_{k}} f(\cdot, k)$ for $n, k \in \omega$. Now our assertion follows by application of 3.4 and 3.6.

The assertion of Proposition 3.9 below shows that the result of [DDM, Lemma 4.2] is fully recovered for the spaces $C_{F}^{*}$. The proof of 3.9 requires a particular case of the following general fact.

3.8. Proposition. Let $X$ be a separable metrizable space such that $X=$ $\bigcup_{n=1}^{\infty} X_{n}, X_{n} \in M_{\alpha}, \alpha \geq 2$ and $X_{n} \in A_{\beta}(X)$, where $\beta<\alpha$. Then $X \in M_{\alpha}$.

Proof. Let $Y$ be a metrizable compactification of $X$ and let $Y_{n} \in A_{\beta}$ be such that $Y_{n} \cap X=X_{n}$. Consider $Z=\bigcup_{n=1}^{\infty} Y_{n} \in A_{\beta} \subseteq M_{\alpha}$. We have $Y_{n} \backslash X_{n} \in A_{\alpha}$; hence, $\bigcup_{n=1}^{\infty}\left(Y_{n} \backslash X_{n}\right) \in A_{\alpha}$ and $X=Z \backslash \bigcup_{n=1}^{\infty}\left(Y_{n} \backslash X_{n}\right) \in$ $M_{\alpha}$.

3.9. Proposition. Let $F$ be a filter on $\omega, \alpha$ be a countable ordinal, $\alpha \geq 1$, and let $n \in \omega$. We have:

(a) if $F \in \mathcal{A}_{\alpha} \backslash \mathcal{M}_{\alpha}$, then $C_{F}^{*} \in \mathcal{M}_{\alpha+1} \backslash \mathcal{A}_{\alpha+1}$,

(b) if $F \in \mathcal{M}_{\alpha} \backslash \mathcal{A}_{\alpha}$, then $C_{F}^{*} \in \mathcal{M}_{\alpha} \backslash \mathcal{A}_{\alpha}$,

(c) if $F \in \mathcal{M}_{\alpha} \cap \mathcal{A}_{\alpha} \backslash \bigcup_{\beta<\alpha}\left(\mathcal{A}_{\beta} \cup \mathcal{M}_{\beta}\right)$, then $C_{F}^{*} \in \mathcal{M}_{\alpha} \backslash \mathcal{A}_{\alpha}$,

(d) if $F \in \mathcal{P}_{n}$, then $C_{F}^{*} \in \mathcal{P}_{n}$.

Proof. The proof of the second part of 3.4 yields that whenever $F \in$ $\mathcal{M}_{\alpha}, C_{F} \cap[-n, n]^{\omega} \in \mathcal{M}_{\alpha}$ for $n \geq 1$. Since $C_{F}^{*}=\bigcup_{n=1}^{\infty}\left(C_{F} \cap[-n, n]^{\omega}\right)$ and each $C_{F} \cap[-n, n]^{\omega}$ is closed in $C_{F}^{*}$, it follows from 3.8 that $C_{F}^{*} \in$ $\mathcal{M}_{\alpha}$ (observe that here $\alpha \geq 2$ since there are no filters of class $\mathcal{M}_{1}$ ). If additionally $F \notin \mathcal{A}_{\alpha}$ then, by $3.3, C_{F}^{*} \in \mathcal{M}_{\alpha} \backslash \mathcal{A}_{\alpha}$; hence (b) follows. Assume $F \in \mathcal{M}_{\alpha} \cap \mathcal{A}_{\alpha} \backslash \bigcup_{\beta<\alpha}\left(\mathcal{A}_{\beta} \cup \mathcal{M}_{\beta}\right)$. Consider the decomposition $F=G \times H$ of $F$ into filters $G$ and $H$ described in the proof of 3.3 (obviously, being Borel, $F$ is not an ultrafilter). We may assume that $G \in \mathcal{M}_{\alpha} \cap \mathcal{A}_{\alpha} \backslash \bigcup_{\beta<\alpha} \mathcal{M}_{\beta}$ (if not, $H$ has this property). Recall that $\Sigma=\left\{\left(x_{n}\right) \in \mathbb{R}^{\omega} \mid\left(x_{n}\right)\right.$ is bounded $\}$. Applying [DMM, Lemma 4.2(3)], we find that $c_{G} \in \mathcal{M}_{\alpha} \backslash \mathcal{A}_{\alpha}$. Since $c_{G}^{*}=c_{G} \cap \Sigma$ and $c_{G}$ is homeomorphic to $c_{G}^{*} \cap(-1,1)^{\omega}$, we conclude that $c_{G}^{*} \in \mathcal{M}_{\alpha} \backslash \mathcal{A}_{\alpha}$. Finally, since $C_{F}^{*}$ contains $c_{G}^{*}$ as a closed set, it follows that $C_{F}^{*} \in \mathcal{M}_{\alpha} \backslash \mathcal{A}_{\alpha}$; this shows (c).

To get (a), suppose $F \in \mathcal{A}_{\alpha} \backslash \mathcal{M}_{\alpha}$. Since $C_{F}^{*}=C_{F} \cap \Sigma, 3.4$ (a) yields $C_{F}^{*} \in \mathcal{M}_{\alpha+1}$. Considering, as previously, $F=G \times H$, we may assume $G \in \mathcal{A}_{\alpha} \backslash \mathcal{M}_{\alpha}$. By [DDM, Lemma 4.2(4)], $c_{G} \in \mathcal{M}_{\alpha+1} \backslash \mathcal{A}_{\alpha+1}$. As before, this implies that $c_{G}^{*} \in \mathcal{M}_{\alpha+1} \backslash \mathcal{A}_{\alpha+1}$ and consequently $C_{F}^{*} \in \mathcal{M}_{\alpha+1} \backslash \mathcal{A}_{\alpha+1}$.

The assertion (d) follows from 3.4(c) and the fact that $C_{F}^{*}=C_{F} \cap \Sigma$.

We now provide a counterpart of [DMM, Lemma 4.3]. Let $X$ be a countable space. For every accumulation point $a$ of $X$ we consider the filter $F_{a}$ 
on $X \backslash\{a\}$ consisting of all neighborhoods of $a$; hence

$$
F_{a}=\left\{Y \in 2^{X \backslash\{a\}} \mid a \in \operatorname{Int}(Y \cup\{a\})\right\} .
$$

3.10. Lemma. Let $X$ be a countable space and $A \subseteq X$ be a dense set. If there exists a countable ordinal $\alpha, \alpha \geq 1$ (resp., an integer $n \in \omega$ ) such that for every accumulation point $a \in X$ the filter $F_{a}$ is in $\mathcal{A}_{\alpha} \cup \mathcal{M}_{\alpha}$ (resp., $\left.F_{a} \in \mathcal{P}_{n}\right)$, then $C_{A}(X) \in \mathcal{M}_{\alpha+1}\left(\right.$ resp.,$\left.C_{A}(X) \in \mathcal{P}_{n}\right)$.

Proof (cf. [DMM, Lemma 4.3]). Let $a$ be an accumulation point of $X$. Define a filter on $A$ by letting

$$
G_{a}=\left\{Y \cap A \mid Y \in F_{a}\right\} .
$$

The filter $G_{a}$ is homeomorphic to

$$
\left\{Y \in F_{a} \mid X \backslash(A \cup\{a\}) \subseteq Y\right\},
$$

a closed subset of $F_{a}$. Therefore $G_{a} \in \mathcal{A}_{a} \cup \mathcal{M}_{\alpha}$ (resp., $G_{a} \in \mathcal{P}_{n}$ ). Let $X_{a}$ be the space $A \cup\{a\}$ topologized by isolating the points of $A \backslash\{a\}$ and using the family $\left\{Y \cup\{a\} \mid Y \in G_{a}\right\}$ as a neighborhood base at $a$ (it may happen that $a \in A$ ). Then either $C_{A}\left(X_{a}\right)$ is isomorphic to $c_{G_{a}}$ (in case $a \in A$ ), or else $C_{A}\left(X_{\alpha}\right)$ is isomorphic to $C_{G_{\alpha}}$ (in case $a \notin A$ ). From Proposition 3.4 and [DMM, Lemma 4.2] (see also [CDM, Corollary 5.3(d)]) it follows that in both cases $C_{A}\left(X_{a}\right) \in \mathcal{M}_{\alpha+1}$ (resp., $C_{A}\left(X_{a}\right) \in \mathcal{P}_{n}$ ). Now, 3.10 is a consequence of the following observation:

$$
C_{A}(X)=\bigcap\left\{C_{A}\left(X_{a}\right) \mid a \text { is an accumulation point of } X\right\} .
$$

3.11. R e m ar k. Let $X_{\alpha}$ be the space from Proposition 2.6. Then $C_{\mathrm{p}}\left(X_{\alpha}\right)$ $\in F_{\sigma \delta}$. Moreover, according to Lemma 3.10, there exists $a \in X_{\alpha}$ such that $F_{a} \notin \bigcup_{\beta<\alpha} \mathcal{A}_{\beta} \cup \mathcal{M}_{\beta}$; in fact, one can show that $a=x_{\alpha}$ has this property. (As shown in [DMM, Corollary 3.6] the filters $F_{a}$ are analytic provided $C_{\mathrm{p}}(X)$ is analytic.) Observe that the filter $F_{a}$ has a base which is of the $F_{\sigma \delta}$-type. Namely, the filter base $\{Y \subset X \backslash\{a\} \mid Y \cup\{a\}$ is a clopen subset of $X$ containing $a\}$ is homeomorphic to

$$
\left\{f \in C_{\mathrm{p}}\left(X_{\alpha}\right) \cap\{0,1\}^{X_{\alpha}} \mid f(a)=1\right\},
$$

a closed subset of $C_{\mathrm{p}}\left(X_{\alpha}\right)$.

4. $Z_{\sigma}$-property of $C_{A}(X)$. We recall that a closed subset $A$ of an absolute neighborhood retract $M$ is a $Z$-set if every map $f: K \rightarrow M$ of a compactum $K$ into $M$ can be approximated by maps $\bar{f}: K \rightarrow M \backslash A$. A space which is a countable union of its own $Z$-sets is called a $Z_{\sigma^{-}}$-space. Obviously, every $Z_{\sigma}$-space is of the first category. It is a well-known fact that a dense convex subset of a convex $Z_{\sigma}$-space is itself a $Z_{\sigma}$-space. Since $C_{A}(X)$ is dense in $\mathbb{R}^{A}$ and since $\Sigma=\left\{\left(x_{n}\right) \in \mathbb{R}^{\omega} \mid\left(x_{n}\right)\right.$ is bounded $\}$ is a $Z_{\sigma}$-space, we have: 
4.1. Lemma. Let $X$ be a space and let $A$ be a countable dense subset of $X$.

(a) The space $C_{A}^{*}(X)$ is a $Z_{\sigma}$-space.

(b) If $X$ is compact, then $C_{A}(X)$ is a $Z_{\sigma}$-space.

4.2. Proposition. Let $X$ be a countable nondiscrete space and let $A$ be a dense subset of $X$. If $C_{A}(X)$ is analytic, then $C_{A}(X)$ and $C_{\mathrm{p}}(X)$ are $Z_{\sigma}$-spaces.

Let us recall that a filter $F$ on a countable set $T$ is of the first category if $F$ belongs to the $\sigma$-field generated by the open sets and the first category sets of $2^{T}$. We need the following fact.

4.3. Lemma. Let $F$ be a first category filter on a countable set $T$. Then for every $0<r \leq \infty$ the space $C_{F} \cap[-r, r]^{\omega}$ is a $Z_{\sigma}$-space.

Proof. By a result of Talagrand [Ta, Theorem 2.1] there exists a sequence of pairwise disjoint finite sets $A_{n} \subset T$ such that every $A \in F$ meets all but finitely many $A_{n}, n \in \mathbb{N}$.

For finite $r$, let

$$
X_{n}=\left\{f \in C_{F} \cap[-r, r]^{\omega} \mid \forall(k>n) \exists\left(m \in A_{k}\right)(|f(m)| \leq 2 r / 3)\right\}
$$

and

$$
Y_{n}=\left\{f \in C_{F} \cap[-r, r]^{\omega} \mid \forall(k>n) \exists\left(m \in A_{k}\right)(|f(m)| \geq r / 3)\right\} .
$$

An argument of the proof of [DMM, Proposition 3.3] shows that each $X_{n}$ and $Y_{n}, n \geq 1$, is a $Z$-set. Moreover, $C_{F} \cap[-r, r]^{\omega}=\bigcup_{n=1}^{\infty} X_{n} \cup Y_{n}$.

For $r=\infty$, let

$$
X_{n, l}=\left\{f \in C_{F} \mid \forall(k>n) \exists\left(m \in A_{k}\right)(|f(m)| \leq l)\right\} .
$$

As previously, each $X_{n, l}$ is a $Z$-set in $C_{F}$ and $C_{F}=\bigcup_{n, l=1}^{\infty} X_{n, l}$.

Pro of of $4.2 .1^{\circ}$ Assume $A$ has an accumulation point $a \in A$. Consider

$$
E=\left\{f \in C_{A}(X) \cap\{0,1\}^{A} \mid f(a)=1\right\},
$$

a closed subset of $C_{A}(X)$. The space $E$ can be identified with $F_{a}=\{U \cap A \mid U$ is clopen and $a \in U\}$. Let $F$ be the filter on $A \backslash\{a\}$ generated by $F_{a}$, i.e.,

$$
F=\left\{B \subset A \backslash\{a\} \mid \exists(f \in E)\left(f^{-1}(\{1\}) \subset B \cup\{a\}\right)\right\} .
$$

By an argument of [DMM, Corollary 3.6], $F$ is an analytic filter and consequently it belongs to the $\sigma$-field generated by the open sets and the first category sets of $2^{A \backslash\{a\}}$. By $4.3, C_{F}$ is a $Z_{\sigma}$-space. Since $C_{A}(X)$ is a dense linear subspace of $C_{F} \times \mathbb{R} \subset \mathbb{R}^{A \backslash\{a\}} \times \mathbb{R}=\mathbb{R}^{A}, C_{A}(X)$ is a $Z_{\sigma}$-space.

$2^{\circ}$ Assume $A$ is discrete. For every $x \in X \backslash A$, let $F_{x}=\{U \cap A \mid x \in \operatorname{Int} U\}$ be a filter of neighborhoods of $x$.

Claim. There exists $x_{0} \in X \backslash A$ such that $F_{x_{0}}$ is of the first category. 
Suppose $F_{x}$ is of the second category for every $x \in X \backslash A$. By a result of Talagrand [Ta, Proposition 23], the filter

$$
F=\bigcap\left\{F_{x} \mid x \in X \backslash A\right\}
$$

is also of the second category. We have $F=\{U \cap A \mid \forall(x \in X \backslash A)(x \in$ Int $U)\}=\{U \cap A \mid X \backslash A \subset \operatorname{Int} U\}$, a filter of neighborhoods of $X \backslash A$. Let $A=\left\{a_{n} \mid n \in \omega\right\}$ and set

$$
S=\left\{f \in C_{A}(X) \mid \forall(n \in \omega)\left(f\left(a_{n}\right)=0 \text { or } f\left(a_{n}\right)=n+1\right)\right\} .
$$

It is clear that $S$ is a closed subset of $C_{A}(X)$; consequently, $S$ is analytic. The map $f \rightarrow f^{-1}(\{0\})$ establishes a homeomorphism of $S$ onto $F$. This yields a contradiction.

Continuing the proof of 4.2 , let $F_{x_{0}}$ be a first category filter. By 4.3, $C_{F_{x_{0}}}$ is a $Z_{\sigma}$-space. Since $C_{A}(X)$ is a linear dense subspace of $C_{F_{x_{0}}}$, the latter is also a $Z_{\sigma}$-space.

Since $C_{\mathrm{p}}(X)$ is a dense linear subspace of a $Z_{\sigma}$-space $C_{A}(X) \times \mathbb{R}^{X \backslash A}$, it itself is a $Z_{\sigma}$-space.

Example 2.11 shows that our Claim fails to hold under a weaker hypothesis that $C_{A}^{*}(X)$ is analytic. Observe also that, in Example 2.11, the suitable spaces $C_{A}(X)$ and $C_{\mathrm{p}}(X)$ are not $Z_{\sigma}$-spaces (they are not first category spaces).

Using the full strength of 4.3 and the fact that dense convex subsets of a convex $Z_{\sigma}$-space are $Z_{\sigma}$-spaces we obtain:

4.4. Re mark. For a countable nondiscrete space $X$ and a dense subset $A$ of $X$ such that $C_{A}(X)$ is analytic, the space $C_{A}(X) \cap[-r, r]^{A}$ is a $Z_{\sigma^{-}}$-space for every $r>0$.

5. Topological identification of $F_{\sigma \delta}$-spaces $C_{A}(X)$. Let us recall that the main result of [DMM] states that for countable nondiscrete $X$ the space $C_{\mathrm{p}}(X)$ is homeomorphic to $\sigma^{\omega}$ provided it is of $F_{\sigma \delta}$-type. In this section we extend this result to some spaces $C_{A}(X)$.

5.1. Proposition. Let $X$ be a space and let $A$ be a countable dense set in $X$. If $C_{A}(X) \in F_{\sigma \delta}$ (resp., $\left.C_{A}^{*}(X) \in F_{\sigma \delta}\right)$ and $A$ contains a nontrivial sequence convergent in $X$, then $C_{A}(X)$ (resp., $\left.C_{A}^{*}(X)\right)$ is homeomorphic to $\sigma^{\omega}$.

Proof. Fix a sequence $\left\{x_{n}\right\}_{n=1}^{\infty} \subset A, \lim x_{n}=x_{0}$ and $x_{n} \neq x_{m}$ for $n \neq m$. Using the fact that $X$ is completely regular, we find a sequence of continuous functions $f_{n}: X \rightarrow[0,1], n>0$, such that $f\left(x_{n}\right)=1$ and 
$f_{n}^{-1}((0,1]) \cap f_{m}^{-1}((0,1])=\emptyset$ for $m \neq n$. For $t=\left(t_{n}\right) \in c_{0}$ define

$$
\varphi(t)=\sum_{n=1}^{\infty} t_{n} f_{2 n} .
$$

Since the series converges uniformly, $\varphi(t) \in C_{\mathrm{p}}(X)$. Clearly, $\varphi$ maps $c_{0}$ injectively into $C_{\mathrm{p}}(X)$. Here is how the image $\varphi\left(c_{0}\right)$ can be linearly complemented in $C_{A}(X)$. For $f \in C_{A}(X)$, define $\left(t_{n}^{f}\right) \in c_{0}$ by letting

$$
t_{n}^{f}=f\left(x_{2 n}\right)-f\left(x_{2 n-1}\right) .
$$

The map $f \rightarrow\left(\left(t_{n}^{f}\right), f-\varphi\left(\left(t_{n}^{f}\right)\right)\right)$ splits $C_{A}(X)$ into $c_{0} \times Z$, where $Z=$ $\left\{f-\varphi\left(\left(t_{n}^{f}\right)\right) \mid f \in C_{A}(X)\right\}$. Since $Z$ is a closed linear subspace of $C_{A}(X)$ and since $c_{0}$ is homeomorphic to $\sigma^{\omega},\left[\mathrm{BM}\right.$, Corollary 5.4] implies that $C_{A}(X)$ is also homeomorphic to $\sigma^{\omega}$.

Note that, in fact, for every $t \in c_{0}$ we have $\varphi(t) \in C_{\mathrm{p}}^{*}(X)$. Therefore, the above splitting determines a similar splitting of $C_{\mathrm{p}}^{*}(X)$. Hence, the assertion for $C_{A}^{*}(X)$ follows as well.

5.2. Corollary. Let $X$ be a nondiscrete Fréchet space. For every countable dense subset $A$ of $X$ such that $C_{A}(X) \in F_{\sigma \delta}\left(\right.$ resp., $\left.C_{A}^{*}(X) \in F_{\sigma \delta}\right)$, the space $C_{A}(X)$ (resp., $\left.C_{A}^{*}(X)\right)$ is homeomorphic to $\sigma^{\omega}$.

The following lemma provides examples of spaces $C_{A}(X)$ that are of $F_{\sigma \delta}$-type.

5.3. Lemma. Let $M$ be a compact space and let $A$ be a countable subset of $C_{\mathrm{p}}(M)$.

(a) If $A$ is dense in a compact set $K \subset \mathbb{R}^{M}$, then $C_{A}(K)$ is an $F_{\sigma \delta}$-set.

(b) For every countable set $X \subset C_{\mathrm{p}}(M)$ such that $A \subseteq X$ and $A$ is dense in $X$, the space $C_{A}(X)$ is an $F_{\sigma \delta}$-set.

Proof. The assertion (a) is that of [Ma1, Theorem 2.3]. Here is a modification of this argument to justify (b). We have $\varphi \in C_{A}(X)$ if and only if for every $f \in X$ and for every $p \in \mathbb{N}$ there exist $n \in \mathbb{N}, k \in \mathbb{N}$ and $m_{1}, \ldots, m_{k} \in$ $M$ so that whenever $g_{1}, g_{2} \in A$ and $\max _{1 \leq i \leq k}\left|g_{j}\left(m_{i}\right)-f\left(m_{i}\right)\right|<1 / n$, $j=1,2$, then $\left|\varphi\left(g_{1}\right)-\varphi\left(g_{2}\right)\right| \leq 1 / p$. By the compactness of $M$ and the fact that $X \subseteq C_{\mathrm{p}}(M)$, each set

$$
\begin{aligned}
\left\{\varphi \in \mathbb{R}^{A} \mid\right. & \exists\left(m_{1}, \ldots, m_{k} \in M\right) \forall\left(g_{1}, g_{2} \in A\right) \\
& {\left.\left[\left(\max _{\substack{1 \leq i \leq k \\
j \in\{1,2\}}}\left|g_{j}\left(m_{i}\right)-f\left(m_{i}\right)\right|<1 / n\right) \Rightarrow\left|\varphi\left(g_{1}\right)-\varphi\left(g_{2}\right)\right| \leq 1 / p\right]\right\} }
\end{aligned}
$$

is closed in $\mathbb{R}^{A}$. Hence, the assertion (b) follows.

5.4. Remark. The compactness of $M$ can be replaced by $\sigma$-compactness. However, the result cannot be extended beyond the class of $\mathcal{A}_{1}$. There exists a countable set $A \subset C_{\mathrm{p}}(P)$, where $P$ is the space of irrationals, so 
that $C_{\mathrm{p}}(A)$ is non-Borel. Namely, by [LvMP, Theorem 4.1] there exists a countable regular space $A$ (with exactly one nonisolated point) such that $C_{\mathrm{p}}(A)$ is analytic and non-Borel. Write $Y=C_{\mathrm{p}}(A)$ and take any surjection $\alpha: P \rightarrow Y$. Then $\alpha^{*}$ given by $\alpha^{*}(f)(a)=f(\alpha(a)), f \in C_{\mathrm{p}}(Y)$, defines an injection of $C_{\mathrm{p}}(Y)$ into $C_{\mathrm{p}}(P)$. Since evidently $A$ embeds in $C_{\mathrm{p}}(Y)=$ $C_{\mathrm{p}}\left(C_{\mathrm{p}}(A)\right), A$ embeds in $C_{\mathrm{p}}(P)$.

Recall that every compact $K$ consisting of first Baire class functions (i.e., a Rosenthal compactum) is a Fréchet space [BFT, Theorem 3F]. Below we include examples of such spaces $K$.

5.5. ExAmPLE. (a) The Helly space consisting of all nondecreasing functions $f$ of $[0,1]^{[0,1]}$ (cf. [E, Exercise 3.2.E]).

(b) The space of functions from $[0,1]$ into $[0,1]$ of total variation $\leq 1$ in $[0,1]^{[0,1]}$.

(c) The unit ball $\left(B_{E^{* *}}, \omega^{*}\right)$ of the second dual $E^{* *}$ with the weak ${ }^{*}$ topology, where $E$ is a separable Banach space which does not contain an isomorphic copy of $\ell^{\prime}$.

All of the above examples contain countable dense subsets consisting of continuous functions. In this case the estimation of the Borel class of the space $C_{A}(X)$ can be obtained via 5.3(a). Let us describe another example of Rosenthal compactum $X$ for which the function spaces $C_{A}(X)$ is of $F_{\sigma \delta}$-type.

5.6. ExAmple. Let $X$ be the well-known two-arrow space, i.e., $X=$ $[0,1] \times\{0,1\}$ with the order topology given by the lexicographic order

$$
\left(t_{1}, \varepsilon_{1}\right) \prec\left(t_{2}, \varepsilon_{2}\right) \quad \text { if } t_{1}<t_{2} \text { or }\left(t_{1}=t_{2} \text { and } \varepsilon_{1}<\varepsilon_{2}\right)
$$

(cf. [E, Exercise 3.10C]). (It is clear that $X$ is separable and first countable. Since $X$ can be identified with the space of nondecreasing functions $f$ : $[0,1] \rightarrow\{0,1\}$, it is easily seen that $X$ is compact.) In the lemma below we evaluate the Borel class of the space $C_{A}(X)$.

5.7. Lemma. For every countable dense set $A \subset X$ the space $C_{A}(X)$ is an $F_{\sigma \delta}$-set.

Proof. For $x \in X$, let $\left\{U_{x, n}\right\}_{n \in \omega}$ be a base of neighborhoods of the point $x$. Using the fact that $X$ is first countable, we see that $f \in C_{A}(X)$ if and only if

(*) for every sequence $\left\{x_{n}\right\}_{n=1}^{\infty} \subset A, x_{n} \neq x, n \geq 1$, with $\lim x_{n}=x \in$ $X$, the $\operatorname{limit} \lim f\left(x_{n}\right)$ exists, and for given $x \in A$ and a sequence $\left\{x_{n}\right\}_{n=1}^{\infty} \subset A$ with $\lim x_{n}=x$ we have $\lim f\left(x_{n}\right)=f(x)$.

Obviously, the second part of $(*)$ holds if and only if for every $x \in A$ and every $m \geq 1$ there exists $n \in \omega$ such that whenever $y \in A \cap U_{x, n}$ then $|f(x)-f(y)| \leq 1 / m$. 
For $f \in \mathbb{R}^{A}$ consider the following property:

$$
\begin{aligned}
& \forall(m \geq 1) \exists(n \geq 1) \forall\left(x_{1} \prec \ldots \prec x_{n} \text { and } x_{j} \in A, 1 \leq j \leq n\right) \\
& \exists(1 \leq i<n)\left(\left|f\left(x_{i}\right)-f\left(x_{i+1}\right)\right| \leq 1 / m\right) .
\end{aligned}
$$

We will show that $(* *)$ holds for $f \in C_{A}(X)$ and that $(* *)$ implies the first part of $(*)$.

Suppose $f \in C_{A}(X)$. Then for every $m \geq 1$ there exists a finite open cover $\left\{U_{1}, \ldots, U_{n}\right\}$ of $X$ such that $\operatorname{diam}\left(f\left(U_{j} \cap A\right)\right)<1 / m, 1 \leq j \leq n$. We may assume that $U_{j}$ is an interval with respect to the order $\prec, 1 \leq j \leq n$. Consider $x_{j} \in A, 1 \leq j \leq n+1$, with $x_{1} \prec \ldots \prec x_{n+1}$. There exist $i, k$, $1 \leq i<k \leq n+1$, and $j, 1 \leq j \leq n$, such that $x_{i}, x_{k} \in U_{j}$. It follows that $x_{i+1} \in U_{j}$. Hence $\left|f\left(x_{i}\right)-f\left(x_{i+1}\right)\right|<1 / m$ and $(* *)$ is satisfied.

Now, suppose $(* *)$ is satisfied. We will show that for every $x \in X$ and $\left\{x_{n}\right\}_{n=1}^{\infty} \subset A, x_{n} \neq x, n \geq 1$, and such that $\lim x_{n}=x, \lim f\left(x_{n}\right)$ exists. Assume, to the contrary, that $\lim f\left(x_{n}\right)$ does not exist. Then there exist $m \geq$ 1 and an increasing (if $x=(t, 0)$ for some $t \in[0,1]$ ) or decreasing (for $x$ of the form $(t, 1))$ subsequence $\left\{x_{n_{k}}\right\}$ of $\left\{x_{n}\right\}$ such that $\left|f\left(x_{n_{k}}\right)-f\left(x_{n_{k+1}}\right)\right| \geq 1 / m$. This clearly contradicts $(* *)$.

It is now clear that $f \in C_{A}(X)$ if and only if

$$
\begin{aligned}
& {\left[\forall(m \geq 1) \exists(n \geq 1) \forall\left(x_{1} \prec \ldots \prec x_{n} \text { and } x_{j} \in A, 1 \leq j \leq n\right)\right.} \\
& \left.\exists(1 \leq i<n)\left(\left|f\left(x_{i}\right)-f\left(x_{i+1}\right)\right| \leq 1 / m\right)\right] \& \\
& {\left[\forall(x \in A) \forall(m \geq 1) \exists(n \in \omega) \forall\left(y \in A \cap U_{x, n}\right)(|f(x)-f(y)| \leq 1 / m)\right] .}
\end{aligned}
$$

The formula (***) shows that the space $C_{A}(X)$ is an $F_{\sigma \delta}$-set.

5.8. Corollary. For each space $X$ from 5.5 (resp., for the space $X$ from 5.6) and every countable set $A$ consisting of continuous functions and dense in $X$ (resp., every countable dense subset $A$ of $X$ ), the spaces $C_{A}(X)$ and $C_{A}^{*}(X)$ are homeomorphic to $\sigma^{\omega}$.

We can also apply 5.1 and 5.3 for the spaces which lack the Fréchet property.

5.9. ExAmple. Let $A$ be the set of all continuous functions from the Cantor set $2^{\omega}$ into $\{0,1\}$ and let $B$ be a countable norm dense subset of the space of continuous functions from $2^{\omega}$ into $[0,1]$. Obviously $A$ and $B$ are countable dense subsets of the products $\{0,1\}^{2^{\omega}}$ and $[0,1]^{2^{\omega}}$, respectively. By Lemma 5.3(a) the spaces $C_{A}\left(\{0,1\}^{2^{\omega}}\right)$ and $C_{B}\left([0,1]^{2^{\omega}}\right)$ are $F_{\sigma \delta}$-sets. Since $A$ and $B$ contain nontrivial convergent sequences, from Proposition 5.1 it follows that $C_{A}\left(\{0,1\}^{2^{\omega}}\right)$ and $C_{B}\left([0,1]^{2^{\omega}}\right)$ are homeomorphic to $\sigma^{\omega}$. Let us point out that 1.1 implies that it is also possible to find countable dense subsets $C \subseteq\{0,1\}^{2^{\omega}}$ and $D \subseteq[0,1]^{2^{\omega}}$ such that the spaces $C_{C}\left(\{0,1\}^{2^{\omega}}\right)$ and $C_{D}\left([0,1]^{2 \omega}\right)$ are not analytic. 
The following result generalizes the main result of [DMM].

5.10. Theorem. Let $X$ be a nondiscrete countable space and let $A$ be a dense subset of $X$ such that $C_{A}(X) \in F_{\sigma \delta}$. Then $C_{A}(X)$ and $C_{A}^{*}(X)$ are homeomorphic to $\sigma^{\omega}$.

5.11. Remark. Let us point out that there exist spaces $X$ satisfying the assumptions of Theorem 5.10 which are not Fréchet spaces (compare 5.10 and 5.2). For example one can easily check that for the filter

$$
F=\left\{A \subset \mathbb{N} \mid \sum_{n \in \mathbb{N} \backslash A} 1 / n<\infty\right\}=\bigcup_{k \in \mathbb{N}}\left\{A \subset \mathbb{N} \mid \sum_{n \in \mathbb{N} \backslash A} 1 / n \leq k\right\} \in F_{\sigma}
$$

the corresponding space $\mathbb{N}_{F}$ does not contain any nontrivial convergent sequence. From Proposition 3.4 and [DMM, Lemma 4.2] it follows that both spaces $C_{\mathrm{p}}\left(\mathbb{N}_{F}\right)$ and $C_{\mathbb{N}}\left(\mathbb{N}_{F}\right)$ are $F_{\sigma \delta}$-sets. The space from [DMM, Example 7.1] also has similar properties.

The proofs of Theorem 5.10 and a few of our next results on the topological identification of $C_{A}(X)$ spaces rely more directly on the absorbing set method than the proof of 5.1. However, we decided to avoid presenting the details of this method. Instead, we formulate two consequences of this method which we will employ.

We say that a space $Y$ is $\mathcal{M}_{\alpha}$-universal, where $\alpha$ is a countable ordinal, $\alpha \geq 1$, (resp., $\mathcal{P}_{n}$-universal, $n \in \omega$ ) if every $A \in \mathcal{M}_{\alpha}$ (resp., $A \in \mathcal{P}_{n}$ ) embeds onto a closed subset of $Y$. We will make use of the $\mathcal{M}_{\alpha}$-universal space $\Omega_{\alpha}$, $\alpha \geq 1$, and the $\mathcal{P}_{n}$-universal space $\Pi_{n}, n \geq 1$; these spaces are known as $\mathcal{M}_{\alpha}$-absorbing and $\mathcal{P}_{n}$-absorbing sets and were constructed in $[\mathrm{BM}]$ and $[\mathrm{C}]$, respectively. We have $\Omega_{\alpha} \in \mathcal{M}_{\alpha} \backslash \mathcal{A}_{\alpha}, \alpha \geq 2$, and $\Pi_{n} \in \mathcal{P}_{n} \backslash \bigcup_{k<n} \mathcal{P}_{k}, n \geq 1$. Obviously, $Y$ is $\mathcal{M}_{\alpha}$-universal (resp., $\mathcal{P}_{n}$-universal) if and only if $\Omega_{\alpha}$ (resp., $\Pi_{n}$ ) embeds onto a closed subset of $Y$. We just have $\Omega_{2}=\sigma^{\omega}$.

5.12. Proposition. Let $Y$ be a linear dense subspace of $\mathbb{R}^{\omega}, \alpha$ be a countable ordinal and $n \in \omega$. Assume that

(i) $Y$ is homeomorphic to $Y \times \mathbb{R}^{\omega}$,

(ii) $Y$ is a $Z_{\sigma}$-space,

(iii) $Y$ is $\mathcal{M}_{\alpha}$-universal and $Y \in \mathcal{M}_{\alpha}, \alpha \geq 2$ (resp., $Y$ is $\mathcal{P}_{n}$-universal and $\left.Y \in \mathcal{P}_{n}, n \geq 1\right)$.

Then $Y$ is homeomorphic to $\Omega_{\alpha}$ (resp., $\left.\Pi_{n}\right)$.

Pr o of. Apply [CDM, Theorem 3.1] and results of [BM].

We will also need the following specific fact which is a slight modification of a particular case of [DMM, Lemma 5.2] (for the proof, see also [CD, Proposition 3.6]). 
5.13. Lemma. Let $X_{i}$ and $Y_{i}$ be dense linear subspaces of $\mathbb{R}^{\omega}, Y_{i} \subseteq X_{i}$, $i \in \mathbb{N}$. Assume that

(iii) $^{\prime} \sigma^{\omega}$ is embeddable in $Y_{i}$ as a closed subset of $X_{i}, i \geq 1$.

Then every $Z_{\sigma}$-space $X$ which is an absolute $F_{\sigma \delta}$-set and satisfies

$$
\left\{\left(x_{i}\right) \in \prod_{i=1}^{\infty} Y_{i} \mid x_{i}=0 \text { a.e. }\right\} \subseteq X \subseteq \prod_{i=1}^{\infty} X_{i}
$$

is homeomorphic to $\sigma^{\omega}$.

Now we are ready to provide the proof of 5.10. First we settle the case of $C_{F}$ and $C_{F}^{*}$ spaces.

5.14. Proposition. Let $F$ be a filter on $\omega$. If $C_{F} \in F_{\sigma \delta}$, then $C_{F}$ is homeomorphic to $\sigma^{\omega}$.

Proof. For the Fréchet filter $F_{0}$, we have $C_{F_{0}}=c$; according to [DM, Theorem 4.2], $C_{F_{0}}$ is homeomorphic to $\sigma^{\omega}$.

Assume $F$ is not the Fréchet filter. By 3.2, $C_{F}$ is homeomorphic to $C_{F} \times \mathbb{R}^{\omega}$, and in view of 3.3 and $4.3, C_{F}$ is a $Z_{\sigma}$-space. Now, Proposition 5.12 is applicable (the case of $\alpha=2$ ) provided $C_{F}$ contains a closed copy of $\sigma^{\omega}$. This is shown in the lemma below.

5.15. Lemma. If $F$ is a first category filter on $\omega$, then $c_{F}$ (resp., $\left.c_{F}^{*}\right)$ contains a copy of $\sigma^{\omega}$ that is closed in $C_{F}$ (resp., in $\left.C_{F}^{*}\right)$.

Pro of. By [Ta, Theorem 2.1] there exists a sequence of pairwise disjoint finite sets $A_{n} \subset \omega$ such that every $A \in F$ meets all but finitely many $A_{n}$, $n \geq 1$. Let $N_{1}=\bigcup_{k=1}^{\infty} A_{2 k}$ and $N_{2}=\omega \backslash N_{1}$. Define $E=\left\{f \in C_{F} \mid\left(f \mid N_{2}\right.\right.$ $=0)$ and $\forall(k \in \mathbb{N})\left(f\right.$ is constant on $\left.\left.A_{2 k}\right)\right\}$, a subset of $c_{F}$ closed in $C_{F}$. We see that $E$ is isomorphic to $c_{0}$ and our assertion for $C_{F}$ follows. The same proof works for $C_{F}^{*}$.

In view of 3.9, the following result is a counterpart of 5.14 for $C_{F}^{*}$ spaces.

5.16. Proposition. If $F$ is an $F_{\sigma \delta}$-filter on $\omega$, then $C_{F}^{*}$ is homeomorphic to $\sigma^{\omega}$

Pro of of 5.16. Applying [DMM, Proposition 2.4], we decompose $\omega$ into infinite sets $N_{i}, i \in \mathbb{N}$, such that $F_{i}=\left\{A \cap N_{i} \mid A \in F\right\}$ is an $F_{\sigma \delta}$-filter on $N_{i}$. For the natural isomorphism $h: \mathbb{R}^{\omega} \rightarrow \prod_{i=1}^{\infty} \mathbb{R}^{N_{i}}$ we have

$$
\left\{\left(x_{i}\right) \in \prod_{i=1}^{\infty} c_{F_{i}}^{*} \mid x_{i}=0 \text { a.e. }\right\} \subset h\left(C_{F}^{*}\right) \subset \prod_{i=1}^{\infty} C_{F_{i}}^{*} .
$$

By 4.1 and 3.9 , the space $C_{F}^{*}$ (and hence $h\left(C_{F}^{*}\right)$ ) is a $Z_{\sigma}$-space and is an absolute $F_{\sigma \delta^{-}}$-set. Now, 5.13 is applicable with $X=h\left(C_{F}^{*}\right), X_{i}=C_{F}^{*}$ and 
$Y_{i}=c_{F_{i}}^{*}$ because, according to $5.15, c_{F_{i}}^{*}$ contains a copy of $\sigma^{\omega}$ which is closed in $C_{F_{i}}^{*}, i \geq 1$.

Proof of 5.10. According to [DMM, Proposition 6.1] either

(1) there exists $Y \subseteq X$ such that $Y$ is clopen with exactly one nonisolated point, or

(2) $X=\bigcup_{n \in \omega} X_{n}$, where each $X_{n}$ is a nondiscrete clopen subset of $X$ and $X_{n} \cap X_{m}=\emptyset$ for $n \neq m$.

If (1) happens, then $C_{A}(X)$ is homeomorphic to the product $C_{A \cap Y}(Y) \times$ $C_{A \backslash Y}(X \backslash Y)$. Since $Y$ can be identified with a space $\mathbb{N}_{F}$ for some filter $F$, by 5.14 and [DMM, Theorem 1.1], $C_{A \cap Y}(Y)$ is homeomorphic to $\sigma^{\omega}$. By [BM, Corollary 5.4], $C_{A}(X)$ is also homeomorphic to $\sigma^{\omega}$. With the help of 5.16 , the same argument yields the case of $C_{A}^{*}(X)$ (here we need the fact that $F$ is an $F_{\sigma \delta}$ filter, which follows from 3.3).

If (2) happens, then $C_{A}(X)$ can be identified with $\prod_{n=1}^{\infty} C_{A \cap X_{n}}\left(X_{n}\right)$. Since $X_{n}$ is not discrete, 4.2 is applicable and each $C_{A \cap X_{n}}\left(X_{n}\right)$ is a $Z_{\sigma}$-space. By [DM, Corollary 2.7], the product $\prod_{n=1}^{\infty} C_{A \cap X_{n}}\left(X_{n}\right)$ is homeomorphic to $\sigma^{\omega}$.

The case of $C_{A}^{*}(X)$ needs some adjustment. We have

$$
\left\{\left(f_{n}\right) \in \prod_{n=1}^{\infty} C_{A \cap X_{n}}^{*}\left(X_{n}\right) \mid f_{n} \equiv 0 \text { a.e. }\right\} \subset C_{A}^{*}(X) \subset \prod_{n=1}^{\infty} C_{A \cap X_{n}}^{*}\left(X_{n}\right) .
$$

According to 4.1 and our assumption $C_{A}^{*}(X)$ is a $Z_{\sigma}$-space which is an absolute $F_{\sigma \delta}$-set. Now, 5.13 is applicable provided we show that each $C_{A \cap X_{n}}^{*}\left(X_{n}\right)$ contains a closed copy of $\sigma^{\omega}$. To show the latter, first we rearrange indices in order to get that each $X_{n}$ satisfies (2). It is now enough to check that whenever $X$ satisfies (2), then $C_{A}^{*}(X)$ contains a closed copy of $\sigma^{\omega}$.

To see this consider $\prod_{n=1}^{\infty} C_{A \cap X_{n}}^{*}\left(X_{n}\right) \cap[-1,1]^{A \cap X_{n}}$, a closed subset of $C_{A}^{*}(X)$. Since $X_{n}$ is nondiscrete, by $4.4, C_{A \cap X_{n}}^{*}\left(X_{n}\right) \cap[-1,1]^{A \cap X_{n}}=$ $C_{A \cap X_{n}}\left(X_{n}\right) \cap[-1,1]^{A \cap X_{n}}$ is a $Z_{\sigma}$-space. Now applying [DMM, Lemma 5.3], we deduce that $\prod_{n=1}^{\infty} C_{A \cap X_{n}}^{*}\left(X_{n}\right) \cap[-1,1]^{A \cap X_{n}}$ contains a closed copy of $\sigma^{\omega}$.

5.17. Remark. Our Theorem 5.10 recovers Theorem 1.1 of [DMM] which was formulated in both absolute and bounded cases. As was kindly brought to our attention by Jan Baars, the proof of the bounded case was not exactly the same as that of the absolute case (as suggested in [DMM]). This is why we included a detailed proof for the case of $C_{A}^{*}(X)$ here.

6. Analytic $C_{A}(X)$ for separable metrizable $X$. Let $X$ be a separable metrizable space. The main result of this section is Theorem 6.2 which contains a complete topological classification of the analytic spaces $C_{A}(X)$ 
and $C_{A}^{*}(X)$. The case where $X$ is discrete is obvious; simply $C_{A}(X)$ and $C_{A}^{*}(X)$ are isomorphic to $\mathbb{R}^{\omega}$ and $\Sigma$, respectively.

We start with the observation that $C_{A}(X)$ is an absolute $F_{\sigma \delta}$-set for every $\sigma$-compact $X$.

6.1. Lemma. Let $X$ be a $\sigma$-compact metrizable space. For every countable dense set $A \subseteq X, C_{A}(X)$ is an absolute $F_{\sigma \delta}$-set.

Proof. Let $X=\bigcup_{n \in \omega} X_{n}$, where $X_{n}$ are compacta, and let $d$ be a metric on $X$. We have

$$
\begin{aligned}
C_{A}(X)=\left\{f \in \mathbb{R}^{A} \mid \forall(n \in \omega) \forall(m \in \mathbb{N}) \exists(l \in \mathbb{N}) \forall(a, b \in A)\right. \\
\\
\left.\quad\left[\left(d(a, b)<1 / l \text { and } \operatorname{dist}\left(a, X_{n}\right)<1 / l\right) \Rightarrow|f(a)-f(b)| \leq 1 / m\right]\right\} .
\end{aligned}
$$

This shows that $C_{A}(X)$ is an absolute $F_{\sigma \delta}$-set.

Here is our main result.

6.2. TheOREM. Let $X$ be a separable metrizable nondiscrete space and let $A$ be a countable dense subset of $X$. The following conditions are equivalent:

(a) $X$ is $\sigma$-compact,

(b) $C_{A}(X)$ is an analytic set,

(c) $C_{A}(X)$ is an absolute $F_{\sigma \delta}$-set,

(d) $C_{A}(X)$ is homeomorphic to $\sigma^{\omega}$,

(e) $C_{A}^{*}(X)$ is an analytic set,

(f) $C_{A}^{*}(X)$ is an absolute $F_{\sigma \delta}$-set,

(g) $C_{A}^{*}(X)$ is homeomorphic to $\sigma^{\omega}$.

Proof. Note that the implications $(\mathrm{d}) \Rightarrow(\mathrm{c}) \Rightarrow(\mathrm{b})$ and $(\mathrm{g}) \Rightarrow(\mathrm{f}) \Rightarrow(\mathrm{e})$ are obvious, $(\mathrm{c}) \Rightarrow(\mathrm{d})$ and $(\mathrm{f}) \Rightarrow(\mathrm{g})$ follow from 5.2 , and $(\mathrm{a}) \Rightarrow(\mathrm{c})$ and $(\mathrm{a}) \Rightarrow(\mathrm{f})$ are consequences of 6.1 . The implication $(\mathrm{b}) \Rightarrow(\mathrm{e})$ follows from the fact that $C_{A}^{*}(X)$ is an $F_{\sigma}$-subset of $C_{A}(X)$.

Here is the proof of the remaining implication (e) $\Rightarrow(\mathrm{a})$. If the space $C_{A}^{*}(X)$ is analytic then its closed subset $S=C_{A}(X) \cap[-1,1]^{A}$ is also analytic. Let $(Y, d)$ be a metric compactification of the space $X$. By $K(Y \backslash X)$ we denote the space of all compact subsets of $Y \backslash X$ equipped with the Vietoris topology. $K(Y \backslash X)$ is a separable metrizable space (cf. [Kur, §17,42]).

Consider the map $\Phi: S \rightarrow K(Y \backslash X)$ defined by

$$
\begin{aligned}
\Phi(f)=\{y \in Y \mid \forall(n \in \mathbb{N}) \exists(a, b \in A)(d(a, y)<1 / n, \\
d(b, y)<1 / n \text { and }|f(a)-f(b)|>1)\},
\end{aligned}
$$

for $f \in S$. One can easily check that $\Phi(f)$ is a closed subset of $Y$ disjoint from $X$, therefore $\Phi$ is well-defined. The map $\Phi$ is onto. Indeed, for given compact $K \subset Y \backslash X$ take a dense subset $\left\{y_{k} \mid k \in \omega\right\}$ of $K$. Let $\left\{\left(k_{i}, n_{i}\right) \mid i \in \omega\right\}$ be an enumeration of $\omega \times \mathbb{N}$. By induction choose points $a_{i}, b_{i} \in A$ such 
that $d\left(a_{i}, y_{k_{i}}\right)<1 / n_{i}, d\left(b_{i}, y_{k_{i}}\right)<1 / n_{i}$ and $\operatorname{dist}\left(a_{i}, K\right)>\operatorname{dist}\left(b_{i}, K\right)>$ $\operatorname{dist}\left(a_{i+1}, K\right)$ for $i \in \omega$. Let $g:(0, \infty) \rightarrow[-1,1]$ be a continuous function such that $g\left(\operatorname{dist}\left(a_{i}, K\right)\right)=1$ and $g\left(\operatorname{dist}\left(b_{i}, K\right)\right)=-1$ for $i \in \omega$. For the function $f: A \rightarrow[-1,1]$ defined by the formula $f(a)=g(\operatorname{dist}(a, K))$ for $a \in A$, we have $f \in S$ and $\Phi(f)=K$.

We will show that $\Phi$ is Borel (in fact of the second class). It is enough to verify that for any given open set $U \subset Y$ the sets

$$
\begin{aligned}
& V_{1}=\{K \in K(Y \backslash X) \mid K \subset U\}, \\
& V_{2}=\{K \in K(Y \backslash X) \mid K \cap U \neq \emptyset\}
\end{aligned}
$$

have Borel inverse images under $\Phi$. We have

$$
\begin{aligned}
& \Phi^{-1}\left(V_{1}\right)=\{f \in S \mid \exists(n \in \mathbb{N}) \forall(a, b \in A) \\
& [(d(a, b)<1 / n \text { and } \operatorname{dist}(a, Y \backslash U)<1 / n) \Rightarrow|f(a)-f(b)| \leq 1]\} \in \mathcal{A}_{1}(S), \\
& \Phi^{-1}\left(V_{2}\right)=\{f \in S \mid \exists(k \in \mathbb{N}) \forall(n \in \mathbb{N}) \exists(a, b \in A) \\
& \quad(d(a, b)<1 / n, \operatorname{dist}(a, Y \backslash U)>1 / k \text { and }|f(a)-f(b)|>1)\} \in \mathcal{A}_{2}(S) .
\end{aligned}
$$

The space $K(Y \backslash X)$, being a Borel image of an analytic space, is also analytic. By [SR2, Theorem 8] (see also [Ch, Theorem 3.3]), $Y \backslash X$ is an absolute $G_{\delta}$-set which means that $X$ is $\sigma$-compact.

Let us mention that the equivalence of the conditions (a), (b) and (c) of Theorem 6.2 was proved by O. Okunev [O, Theorem 2.1]. Also A. Krawczyk (unpublished) obtained a partial result in this direction. It turns out, however, that the main implication between these three conditions, (b) $\Rightarrow(\mathrm{a})$, was implicitly contained in the proof of [Ch, Theorem 3.7]; yet, we were not able to use Chirstensen's argument to prove the (stronger) fact that the analyticity of $C_{A}^{*}(X)$ implies the $\sigma$-compactness of $X$.

7. Spaces $C_{F}$ of arbitrarily high Borel complexity. Let us recall the inductive construction of filters $F_{\alpha}$ ( $\alpha$ odd) and $G_{\alpha}$ ( $\alpha$ even) used in [CDM, Section 8]; here $\alpha$ is a countable ordinal. Fix any filter $F_{1} \in \mathcal{A}_{1}$. Suppose the filters $F_{\beta}$ have been defined for all odd $\beta<\alpha$. If $\alpha-1$ is not a limit ordinal, put $\beta_{n}=\alpha-2, n \geq 1$; if $\alpha-1$ is a limit ordinal, pick $\left(\beta_{n}\right)$ to be a sequence of odd ordinals such that $1<\beta_{n}<\alpha-1$ and $\sup \beta_{n}=\alpha-1$. We let

$$
F_{\alpha}=\mathbb{F} \mathbb{P}\left(2^{\omega}, F_{\beta_{n}}\right)
$$

(see the proof of 2.6 for the definition of the symbol $\mathbb{F P}$; here $X_{n}=2^{\omega}$, $n \in \mathbb{N}$ ). For all even $\alpha, \alpha>0$, define $G_{\alpha}$ as follows. If $\alpha$ is not a limit ordinal, we let $G_{\alpha}=F_{\alpha-1}^{\infty}$. If $\alpha$ is a limit ordinal, pick a sequence $\left(\beta_{n}\right)$ of odd ordinals such that $1<\beta_{n}<\alpha$ and $\sup \beta_{n}=\alpha$, and let $G_{\alpha}=\prod_{n=1}^{\infty} F_{\beta_{n}}$.

7.1. TheOREm. For every odd (resp., even) countable ordinal $\alpha, \alpha>1$, the space $C_{F_{\alpha}}$ (resp., $\left.C_{G_{\alpha}}\right)$ is homeomorphic to $\Omega_{\alpha+1}$ (resp., $\Omega_{\alpha}$ ). 
Proof. By [CDM, Lemma 8.6], for every $\alpha$ we have $F_{\alpha} \in \mathcal{A}_{\alpha}$ (actually, $F_{\alpha} \in \mathcal{A}_{\alpha} \backslash \mathcal{M}_{\alpha}$ ). This, together with 3.4(a) and 3.7, shows that for odd (resp., even) $\alpha, C_{F_{\alpha}} \in \mathcal{M}_{\alpha+1}$ (resp., $C_{G_{\alpha}} \in \mathcal{M}_{\alpha}$ ).

It follows directly from the construction of $F_{\alpha}$ and $G_{\alpha}$ that for $\alpha>1$ the filter $F_{\alpha}$ (resp., $G_{\alpha}$ ) can be identified with the product $F_{\alpha} \times F_{\alpha}$ (resp., $G_{\alpha} \times G_{\alpha}$ ) defined on the union of two disjoint copies of $\omega$. With this identification we see that $C_{F_{\alpha} \times F_{\alpha}}$ (resp., $C_{G_{\alpha} \times G_{\alpha}}$ ) contains $c_{F_{\alpha}}$ (resp., $c_{G_{\alpha}}$ ) as a closed subset (cf. the proof of 3.3). By [CDM, Theorem 8.7], $c_{F_{\alpha}}$ (resp., $c_{G_{\alpha}}$ ) is homeomorphic to $\Omega_{\alpha+1}$ (resp., $\Omega_{\alpha}$ ). This shows that $C_{F_{\alpha} \times F_{\alpha}}$, and hence $C_{F_{\alpha}}$, is $\mathcal{M}_{\alpha+1}$-universal (resp., $c_{G_{\alpha} \times G_{\alpha}}$, and hence $C_{G_{\alpha}}$, is $\mathcal{M}_{\alpha}$-universal). Obviously none of the filters $F_{\alpha}$ and $G_{\alpha}, \alpha>1$, is the Fréchet filter; consequently, 3.2 is applicable to deduce that $C_{F_{\alpha}}$ (resp., $C_{G_{\alpha}}$ ) is homeomorphic to $C_{F_{\alpha}} \times \mathbb{R}^{\omega}$ (resp., $C_{G_{\alpha}} \times \mathbb{R}^{\omega}$ ). By $4.3, C_{F_{\alpha}}$ (resp., $C_{G_{\alpha}}$ ) is a $Z_{\sigma^{-}}$-space. Finally, our assertion follows from 5.12 .

To provide spaces $C_{F}$ homeomorphic to $\Omega_{\alpha}$ for arbitrary $\alpha>1$, we shall make use of filters $F=F_{A}, A \subseteq I^{\omega}$, described in [CDM, Section 9]. In this way, we also find filters $F_{n}$ such that $C_{F_{n}}$ is homeomorphic to $\Pi_{n}, n \geq 1$. First, let us recall the construction of filters $F_{A}$.

Let $d$ be a metric on $I^{\omega}$ bounded by 1 . Fix $Q_{k}$, a finite $(1 / k)$-net in $I^{\omega}$. Assume $\left\{Q_{k}\right\}_{k=1}^{\infty}$ is pairwise disjoint and let $\left\{q_{n}\right\}_{n=1}^{\infty}$ be an enumeration of $\bigcup_{k=1}^{\infty} Q_{k}$. For $k \in \mathbb{N}$ put $N_{k}=\left\{n \in \omega \mid q_{n} \in Q_{k}\right\}$. For every $q \in I^{\omega}$, set

$$
B_{q}=\bigcup_{n=1}^{\infty}\left\{n \in N_{k} \mid d\left(q, q_{n}\right) \leq 2 / k\right\} .
$$

Finally, for $A \subseteq I^{\omega}$ let $F_{A}$ be the filter on $\omega$ generated by the sets of the form

$$
\omega \backslash\left(B_{q_{1}} \cup \ldots \cup B_{q_{n}} \cup S\right),
$$

where $n \in \omega, q_{j} \in A, 1 \leq j \leq n$, and $S$ is a finite set. For $A=I^{\omega}$, we write $F_{A}=F$.

According to [CDM, Proposition 9.2], $F_{A} \in \mathcal{M}_{\alpha}$ (resp., $\mathcal{P}_{n}$ ) whenever $A \in \mathcal{M}_{\alpha}\left(I^{\omega}\right)$ (resp., $A \in \mathcal{P}_{n}\left(I^{\omega}\right)$ ). The following lemma provides a positive answer to 3.4 for filters $F_{A}$.

7.2. Lemma. For every $A \in \mathcal{M}_{\alpha}\left(I^{\omega}\right)$ (resp., $\left.\mathcal{P}_{n}\left(I^{\omega}\right)\right)$, the space $C_{F_{A}}$ belongs to $\mathcal{M}_{\alpha}$ (resp., $\left.\mathcal{P}_{n}\right)$.

Proof. First we extract $j_{k} \in N_{k}, k \in \mathbb{N}$, so that $\left\{j_{k}\right\} \rightarrow \infty$ in $\mathbb{N}_{F_{B}}$ for every $B \subseteq I^{\omega}$. To this end, fix $q_{0} \in I^{\omega}$ and find $j_{k} \in N_{k}$ so that

$$
2 / k<d\left(q_{0}, q_{j_{k}}\right) \leq 4 / k \text {. }
$$

It is clear that for a given $q \in Q$, there exists $k_{0}$ so that $d\left(q, q_{j_{k}}\right)>2 / k$ for $k \geq k_{0}$. It follows that $j_{k} \in \omega \backslash B_{q}$ for $k \geq k_{0}$; and hence $\left\{j_{k}\right\} \rightarrow \infty$ in each $\mathbb{N}_{F_{B}}, B \subseteq I^{\omega}$. 
Recall $C_{F}=C_{I^{\omega}}$. One sees that $f \in C_{F}$ if and only if, given $\varepsilon>0$, all $n$ such that $|f(n)-f(\infty)| \geq \varepsilon$ belong to $B_{q_{1}} \cup \ldots \cup B_{q_{k}} \cup S$ for some $q_{1}, \ldots, q_{k} \in I^{\omega}$ and some finite set $S$. It follows that such an $f \in C_{F}$ belongs to $C_{F_{A}}$ if additionally we may require that none of $q_{1}, \ldots, q_{k}$ belongs to $I^{\omega} \backslash A$. This happens exactly when for every $q \in I^{\omega} \backslash A$ and every $\varepsilon>0$, $\mid f(n)-f\left(\infty \mid<\varepsilon\right.$ for all but finitely many $n \in B_{q}$. Since $\left\{j_{k}\right\} \rightarrow \infty$ in $\mathbb{N}_{F_{A}}$, the above statement is equivalent to requiring that for every $q \in I^{\omega} \backslash A$ and every $\varepsilon>0$ there exists $k_{0} \in \omega$ such that for $k \geq k_{0}$ and for all $n \in N_{k} \cap B_{q}$ we have $\left|f\left(j_{k}\right)-f(n)\right|<\varepsilon$. For every $q \in I^{\omega}$, define

$$
\begin{aligned}
C_{q}=\left\{f \in C_{F} \mid\right. & \forall(\varepsilon>0) \exists\left(k_{0} \in \mathbb{N}\right) \forall\left(k \geq k_{0}\right) \\
& \left.\forall\left(n \in N_{k} \cap B_{q}\right)\left(\left|f\left(j_{k}\right)-f(n)\right|<\varepsilon\right)\right\} .
\end{aligned}
$$

We have

$$
C_{F_{A}}=\left\{f \in C_{F} \mid \forall\left(q \in I^{\omega} \backslash A\right)\left(f \in C_{q}\right)\right\} .
$$

Suppose now that $A \in \mathcal{M}_{\alpha}, \alpha \geq 2$. Then we can write $A=\bigcap_{n=1}^{\infty} B_{n}$, where $B_{n} \in \bigcup_{\beta<\alpha} \mathcal{A}_{\beta}$. We have

$$
\begin{aligned}
C_{F_{A}} & =\left\{f \in C_{F} \mid \forall\left(q \in I^{\omega} \backslash \bigcap_{n=1}^{\infty} B_{n}\right)\left(f \in C_{q}\right)\right\} \\
& =\bigcap_{n=1}^{\infty}\left\{f \in C_{F} \mid \forall\left(q \in I^{\omega} \backslash B_{n}\right)\left(f \in C_{q}\right)\right\}=\bigcap_{n=1}^{\infty} C_{F_{B_{n}}} .
\end{aligned}
$$

Applying [CDM, Proposition 9.2], we obtain $F_{B_{n}} \in \bigcup_{\beta<\alpha} \mathcal{A}_{\beta}$. By 3.4, $C_{F_{B_{n}}} \in \mathcal{M}_{\alpha}$; consequently, $C_{F_{A}} \in \mathcal{M}_{\alpha}$.

The case where $A \in \mathcal{P}_{n}\left(I^{\omega}\right)$ follows from [CDM, Proposition 9.2] and $3.4(\mathrm{c})$.

7.3. Theorem. Let $A \in \mathcal{M}_{\alpha}\left(I^{\omega}\right), \alpha \geq 2$ (resp., $A \in \mathcal{P}_{n}\left(I^{\omega}\right), n \geq 1$ ). If $A$ is $\mathcal{M}_{\alpha}$-universal (resp., $\mathcal{P}_{n}$-universal), then $C_{F_{A}}$ is homeomorphic to $\Omega_{\alpha}$ (resp., $C_{F_{A}}$ is homeomorphic to $\left.\Pi_{n}\right)$. In particular, for $A=\Omega_{\alpha} \subset I^{\omega}$ (resp., $\left.A=\Pi_{n} \subset I^{\omega}\right), C_{F_{\Omega_{\alpha}}}$ (resp., $C_{F_{\Pi_{n}}}$ ) is homeomorphic to $\Omega_{\alpha}$ (resp., $\left.\Pi_{n}\right)$.

Proof. Lemma 7.2 implies that $c_{F_{A}} \in \mathcal{M}_{\alpha}$ (resp., $C_{F_{A}} \in \mathcal{P}_{n}$ ). The statement of [CDM, Proposition 9.1] easily guarantees that $A$ can be embedded onto a closed subset of $C_{F_{A}}$. Consequently, $C_{F_{A}}$ is $\mathcal{M}_{\alpha}$-universal (resp., $\mathcal{P}_{n}$-universal). Since $F_{A}$ is not the Fréchet filter, 3.2 is applicable; and consequently $C_{F_{A}}$ is homeomorphic to $C_{F_{A}} \times \mathbb{R}^{\omega}$. If $A$ is Borel then, by application of [CDM, Proposition 9.1] and 4.3, the space $C_{F_{A}}$ is a $Z_{\sigma}$-space. If $A$ is merely projective, then $C_{F_{A}}$ is also a $Z_{\sigma}$-space because $C_{F_{A}}$ is a dense linear subspace of the linear space $C_{F}\left(\right.$ recall $\left.F=I^{\omega}\right)$. Now, our assertion follows from 5.12 . 


\section{References}

[BP] C. Bessaga and A. Pełczyński, Selected Topics in Infinite-Dimensional Topology, PWN, Warszawa, 1975.

[BM] M. Bestvina and J. Mogilski, Characterizing certain incomplete infinitedimensional retracts, Michigan Math. J. 33, 1986, 291-313.

[BFT] J. Bourgain, D. H. Fremlin and M. Talagrand, Pointwise compact sets of Baire-measurable functions, Amer. J. Math. 100 (1978), 845-886.

[C] R. Cauty, Ensembles absorbants pour les classes projectives, Fund. Math. 143 (1993), 203-206.

[CD] R. Cauty and T. Dobrowolski, Applying coordinate products to the topological identification of normed spaces, Trans. Amer. Math. Soc. 337 (1993), 625-649.

[CDM] R. Cauty, T. Dobrowolski and W. Marciszewski, A contribution to the topological classification of the spaces $C_{\mathrm{p}}(X)$, Fund. Math. 142 (1993), 269-301.

[Ch] J. P. R. Christensen, Topology and Borel Structure, North-Holland, Amsterdam, 1974.

[DGM] T. Dobrowolski, S. P. Gulko and J. Mogilski, Function spaces homeomorphic to the countable product of $\ell_{2}^{f}$, Topology Appl. 34 (1990), 153-160.

[DM] T. Dobrowolski and J. Mogilski, Certain sequence and function spaces homeomorphic to the countable product of $\ell_{2}^{f}$, J. London Math. Soc. 45 (1992), $566-576$.

[DMM] T. Dobrowolski, W. Marciszewski and J. Mogilski, Topological classification of function spaces $C_{p}(X)$ of low Borel complexity, Trans. Amer. Math. Soc. 328 (1991), 307-324.

[En] R. Engelking, General Topology, PWN, Warszawa, 1977.

[Go] G. Godefroy, Compacts de Rosenthal, Pacific J. Math. 91 (1980), 293-306.

[Kur] K. Kuratowski, Topology, I and II, Academic Press and Polish Scientific Publishers, New York and Warszawa, 1966 and 1968.

[Lu] D. Lutzer, On generalized ordered spaces, Dissertationes Math. 89 (1971).

[LvMP] D. Lutzer, J. van Mill and R. Pol, Descriptive complexity of function spaces, Trans. Amer. Math. Soc. 291 (1985), 121-128.

[Ma1] W. Marciszewski, On a classification of pointwise compact sets of the first Baire class functions, Fund. Math. 133 (1989), 195-209.

[Ma2] -, On analytic and coanalytic function spaces $C_{\mathrm{p}}(X)$, Topology Appl. 50 (1993), 241-248.

[vM] J. van Mill, Topological equivalence of certain function spaces, Compositio Math. 63 (1987), 159-188.

[Ne] S. Negrepontis, Banach spaces and topology, in: Handbook of Set-Theoretic Topology, North-Holland, 1984, 1045-1142.

[O] O. G. Okunev, On analyticity in cosmic spaces, Comment. Math. Univ. Carolin. 34 (1993), 185-190.

[P] R. Pol, On weak and pointwise topology in function spaces, preprint 4184, University of Warsaw, 1984.

[SR1] J. Saint Raymond, Fonctions boréliennes sur quotients, Bull. Sci. Math. (2) 100 (1976), 141-147. 
[SR2] J. Saint Raymond, Caractérisation d'espaces polonais, Séminaire Choquet (Initiation à l'analyse) 1971-73, no. 5, 1-10.

[Ta] M. Talagrand, Compacts de fonctions mesurables et filtres non mesurables, Studia Math. 67 (1980), 13-43.

DEPARTMENT OF MATHEMATICS THE UNIVERSITY OF OKLAHOMA NORMAN, OKLAHOMA 73019-0315

U.S.A.

Current address:

DEPARTMENT OF MATHEMATICS PITTSBURG STATE UNIVERSITY PITTSBURG, KANSAS 66762 U.S.A.

E-mail: TDOBROWO@MAIL.PITTSTATE.EDU
INSTITUTE OF MATHEMATICS WARSAW UNIVERSITY

BANACHA 2

02-097 WARSZAWA, POLAND

Current address: FACULTEIT DER WISKUNDE EN INFORMATICA

VRIJE UNIVERSITEIT DE BOELELAAN 1081A 1081 HV AMSTERDAM, THE NETHERLANDS E-mail: WMARCISZ@CS.VU.NL

Received 5, December 1994;

in revised form 28 February 1995 